Otimização de Métodos Analíticos Usando Metodologia de Superfícies De Resposta - Parte I: Variáveis de Processo

Novaes, C. G.; Yamaki, R. T.; de Paula, V. F.; do Nascimento Júnior, B. B.; Barreto, J. A.; Valasques, G. S.; Bezerra, M. A.*

Rev. Virtual Quim., 2017, 9 (3), 1184-1215. Data de publicação na Web: 5 de abril de 2017

$\underline{\text { http://rvq.sbq.org.br }}$

\title{
Optimization of Analytical Methods Using Response Surface Methodology - Part I: Process Variables
}

Abstract: The present paper approaches the application of response surface methodology (RSM) in the optimization of variables for analytical methods development. Some basic principles of this multivariate method are presented and characteristics of the main experimental designs are discussed. Examples of RSM application for optimization of analytical methods is also presented to illustrate the potentialities of these chemometric tools.

Keywords: Response surface methodology; process variables; optimization of analytical methods.

\section{Resumo}

O presente artigo aborda a aplicação de metodologia de superfícies de resposta (MSR) na otimização de variáveis para o desenvolvimento de métodos analíticos. Alguns princípios básicos desse método multivariado são apresentados e as características dos principais planejamentos experimentais são discutidas. Exemplos de aplicações da MSR na otimização de métodos analíticos são também apresentados para ilustrar as potencialidades dessas ferramentas quimiométricas.

Palavras-chave: Metodologia de superfícies de respostas; variáveis de processo; otimização de métodos analíticos.

\footnotetext{
* Universidade Estadual do Sudoeste da Bahia, Departamento de Química e Exatas, Campus de Jequié, Rua José Moreira Sobrinho, s/n, CEP 45206-190 Jequié-BA, Brasil.

M mbezerra@uesb.edu.br

DOI: $\underline{10.21577 / 1984-6835.20170070}$
} 


\title{
Otimização de Métodos Analíticos Usando Metodologia de Superfícies De Resposta - Parte I: Variáveis de Processo
}

\author{
Cleber G. Novaes, Regina T. Yamaki, Vanderlúcia F. de Paula, Baraquízio \\ B. do Nascimento Júnior, Jeferson A. Barreto, Gisseli S. Valasques, \\ Marcos A. Bezerra*
}

Universidade Estadual do Sudoeste da Bahia, Departamento de Química e Exatas, Campus de Jequié, Rua José Moreira Sobrinho, s/n, CEP 45206-190 Jequié-BA, Brasil.

*mbezerra@uesb.edu.br

Recebido em 2 de janeiro de 2017. Aceito para publicação em 29 de março de 2017

1. Introdução

2. Variáveis e respostas em MSR

3. Planejamentos experimentais para modelagem de superfícies de resposta

4. Planejamentos experimentais para ajuste de funções quadráticas

4.1. Planejamento Composto Central

4.2. Planejamento Box-Behnken

4.3. Planejamento Doehlert

5. Ajuste de modelo matemático

5.1. Avaliação do modelo matemático ajustado

6. Determinação das condições ótimas

7. Programas estatísticos usados em planejamento experimental

8. Exemplos da aplicação da MSR na otimização de um método analítico

9. Conclusões

\section{Introdução}

A otimização tem como objetivo melhorar o desempenho de um sistema, de um processo ou de um produto de forma a obter deles o máximo de benefícios possíveis. Em um método analítico, diversas variáveis (também chamadas fatores) podem influenciar na magnitude e na qualidade do sistema estudado. Sendo assim, os métodos analíticos, ao serem desenvolvidos, também necessitam de otimização por meio da investigação de variáveis e dos seus níveis. 0 termo "otimização" tem sido empregado em química analítica para designar um conjunto de experimentos que visam calcular as condições de realização de um método que, ao serem aplicadas, produzem a melhor 
resposta possível garantindo a este, maior sensibilidade e melhores características analíticas. $^{1,2}$

Tradicionalmente, os processos de otimização são realizados pelo monitoramento de um fator por vez e observação de sua influência sobre uma resposta experimental. Neste tipo de otimização, chamado de otimização univariada, enquanto o nível de um fator é alterado, os níveis dos demais são mantidos em um valor constante. Como consequência, esta técnica não possibilita a avaliação dos efeitos de interação entre as variáveis. ${ }^{3}$

Em anos recentes, tem aumentado a utilização de ferramentas quimiométricas multivariadas para a otimização de métodos analíticos, considerando suas vantagens como a redução do número de experimentos, resultando em menor gasto de reagentes e de tempo. Estas ferramentas baseiam-se no desenvolvimento de modelos matemáticos que permitem estabelecer a relevância e a significância estatística dos efeitos dos fatores estudados, bem como avaliar os efeitos de interação entre os mesmos. Havendo efeitos de interação, as condições ótimas estabelecidas pelo método multivariado poderão ser mais confiáveis do que aquelas obtidas utilizando o método univariado. ${ }^{4}$ Desta forma, o procedimento univariado pode falhar quando o efeito de uma ou mais variáveis for dependente dos níveis das outras envolvidas na otimização. Técnicas multivariadas vêm sendo muito utilizadas no desenvolvimento de métodos analíticos por serem mais econômicas e efetivas, além de permitir que as variáveis estudadas sejam otimizadas simultaneamente. ${ }^{5,6}$

Planejamentos fatoriais completos ou fracionários são planejamentos multivariados que baseiam-se na variação de fatores simultaneamente a um limitado número de níveis (normalmente dois) para cada variável. Esses tipos de planejamentos são especialmente importantes no início de um estudo experimental, pois permite realizar triagem de variáveis evidenciando as mais significativas e quantificando suas interações.
Isso é feito com uma grande economia de experimentos e de, consequentemente, tempo, esforços e custos ao contrário da otimização univariada que não possibilita ter uma ideia inicial do número de experimentos que serão realizados. ${ }^{6,65}$

Dentre as diversas técnicas multivariadas de otimização, a metodologia de superfícies de respostas (MSR) vem sendo muito aplicada por causa de sua alta eficiência, poder de modelagem e capacidade de exploração dos sistemas estudados. A MSR pode ser entendida como uma combinação de técnicas de planejamento de experimentos, análise de regressão e métodos de otimização e se baseia no ajuste de modelos matemáticos empíricos aos dados experimentais obtidos segundo uma determinada matriz experimental com o objetivo de descrever o comportamento destes dados e fazer previsões estatisticamente válidas. ${ }^{7}$ Desta forma, a MSR é muito utilizada para quantificar e interpretar as relações entre as respostas e os efeitos dos fatores. ${ }^{8}$

O presente artigo discute $\mathrm{o}$ uso de metodologia de superfícies de resposta na otimização das variáveis que influenciam o desempenho de métodos analíticos. Serão abordados os princípios básicos da MSR, as suas principais matrizes de planejamento, além do ajuste e avaliação da qualidade do modelo usado na descrição das respostas estudadas. A aplicação da MSR na otimização de variáveis de mistura será discutida na Parte II da revisão.

\section{Variáveis e respostas em MSR}

Ao aplicar um procedimento multivariado de otimização como a MSR, deve-se reconhecer dois tipos de variáveis envolvidas: as variáveis independentes (os fatores) e as variáveis dependentes (as respostas). ${ }^{2}$

Para os fatores ou variáveis independentes, é possível estudar alguns de seus diversos níveis em diferentes combinações com os níveis de outras 
variáveis. Os níveis de uma variável são os diferentes valores que esta variável pode assumir. A influência da variável temperatura sobre uma resposta, por exemplo, pode ser investigada nos seguintes níveis: $30,40,50$, 60 e 70 ㄷ. Os níveis mínimos e máximos das variáveis (30 e 70, neste caso) delimitam o domínio experimental do sistema estudado. ${ }^{6}$

Dois tipos de variáveis dependentes podem influenciar a resposta de um sistema químico: as de método e as de mistura. Fatores como tempo, $\mathrm{pH}$, temperatura, massa da amostra, velocidade de agitação, concentrações dos reagentes, etc., são exemplos típicos de variáveis de método. Alterações nos níveis das variáveis de método podem afetar o resultado experimental através de seus efeitos principais e de interação com outras variáveis de método, mas seus níveis podem ser variados de forma independente uns dos outros na aplicação da matriz experimental. Por outro lado, quando variáveis de mistura estão envolvidas em uma otimização (ex: percentagem de solventes na composição da fase móvel em uma análise cromatográfica), o resultado depende da proporção em que esses componentes se encontram e seus níveis não podem ser independentemente variados sem levar em conta os outros componentes. ${ }^{6,16}$

Ao contrário das variáveis independentes, a magnitude da resposta (ou das respostas) depende da combinação entre os níveis dos fatores e se constituem dos valores de propriedades medidas a partir da execução dos experimentos definidos por uma matriz experimental. Respostas típicas são, por exemplo, os sinais analíticos (absorbância, intensidade de emissão, sinal elétrico, ...), recuperação de um analito, resolução entre picos cromatográficos, entre outros.

Uma situação muito comum é a necessidade de se otimizar várias respostas ao mesmo tempo. A otimização de multirespostas pode ser realizada de duas maneiras. $\mathrm{Na}$ primeira abordagem, as superfícies podem ser determinadas para cada resposta separadamente e então analisadas simultaneamente por simples inspeção visual. Neste caso, as diferentes respostas devem apresentar comportamentos similares quando os níveis dos fatores forem mudados. Um exemplo, durante a otimização de um método analítico que envolva uma técnica multielementar (Ex: ICP OES) serão geradas várias respostas com comportamento muito semelhante entre si. No entanto, se o comportamento das respostas estudadas apresentarem certa divergência, mas ainda possuírem uma região comum onde as respostas são consideradas satisfatórias, as condições ótimas podem ser encontradas por superposição das superfícies geradas. ${ }^{9}$ Porém, à medida que a divergência entre as respostas aumenta torna-se mais difícil encontrar uma região de intersecção satisfatória e, em consequência, mudança no nível de um fator pode melhorar uma resposta específica e ter um efeito bastante deletério sobre as outras respostas. ${ }^{12}$

Numa segunda abordagem, as diversas respostas podem ser combinadas em uma única através do uso de uma função matemática. A superfície de resposta obtida permite calcular os valores ótimos em relação a cada variável que satisfaça simultaneamente, e na medida do possível, todas as respostas consideradas.

Uma forma de trabalhar as multirespostas bastante interessante e que está se popularizando é pela aplicação de uma função de desejabilidade. Este tratamento foi proposto por Derringer e Suich em $1980 .{ }^{10}$ Esta metodologia baseia-se na transformação de cada resposta individual em uma escala adimensional de desejabilidades individuais $\left(d_{i}\right)$. A escala de desejabilidades individuais pode variar de 0 (para uma resposta inaceitável) a 1 (para a resposta desejada). Esta transformação torna possível combinar os resultados obtidos para as propriedades medidas em diferentes ordens de grandeza. Com as desejabilidades individuais, torna-se possível obter a desejabilidade global (D), definida como a média geométrica das desejabilidades individuais $\left(\mathrm{d}_{\mathrm{i}}\right)$ de acordo com a equação: 


$$
D=\sqrt[m]{d_{1} d_{2} \ldots d_{m}}
$$

Eq. 1

onde $\mathrm{m}$ é o número de respostas estudadas no processo de otimização. Desta forma, o processo de otimização simultânea é reduzida à simples tarefa de se calcular os níveis das variáveis que maximizem a

$$
\mathrm{d}=\left\{\begin{array}{l}
0 \text { se } y<L \\
\left(\frac{y-L}{T-L}\right)^{s} \text { se } L \leq y \leq T \\
1 \text { se } y>T
\end{array}\right.
$$

onde L é o menor valor aceitável para a resposta e $s$ é o peso. Então, quando $s=1$, a função de desejabilidade é linear e quando $s$ $>1$, uma maior importância é dada aos valores perto do ótimo.

Funções para o cálculo de desejabilidades individuais também podem ser estabelecidas para outras situações como o valor alvo ser um mínimo ou estiver localizado entre um valor mínimo e um valor máximo. A aplicação de função de desejabilidade na otimização de métodos analíticos traz inúmeras vantagens como eficiência, economia e objetividade na otimização de procedimentos com múltiplas respostas. ${ }^{11,12}$

Outros métodos para otimização de multirespostas, diferentes da abordagem de Derringer-Suich, também tem sido propostos. Uma forma de obter uma resposta combinada é realizando-se a divisão de cada resposta individual pela maior resposta observada no conjunto de dados e somandose todos os resultados obtidos para se obter uma única resposta ${ }^{13}$. Bezerra et al. ${ }^{14}$ em uma abordagem diferente, usaram componentes principais para otimizar um procedimento de pré-concentração de seis metais $(\mathrm{Cd}, \mathrm{Cr}, \mathrm{Cu}$, $\mathrm{Mn}, \mathrm{Ni}$ e $\mathrm{Pb}$ ) para posterior determinação por ICP OES. Os comportamentos de cinco desses metais foram altamente correlacionados e puderam ser todos descritos pela primeira componente principal enquanto que o comportamento do $\mathrm{Ni}$ foi descrito pela desejabilidade global.

Há diferentes tipos de transformações possíveis para a obtenção das desejabilidades individuais. Se o valor alvo ( $T$, de target value) para a resposta $y$, por exemplo, for um máximo, a desejablidade individual $\left(\mathrm{d}_{\mathrm{i}}\right)$ é descrita pela seguinte função:

segunda componente principal.

\section{Planejamentos experimentais para modelagem de superfícies de resposta}

Um planejamento experimental baseia-se em um conjunto de experimentos definidos por uma matriz composta por diferentes combinações de níveis das variáveis estudadas e deve atender certos critérios desejáveis como: proporcionar boas estimativas para os coeficientes da função que será ajustada aos dados experimentais exigindo o menor número de experimentos possíveis e, fornecer condições de avaliação dos coeficientes e do modelo matemático usado. ${ }^{2}$

O modelo mais simples que pode ser utilizado em MSR baseia-se em funções lineares e pode ser facilmente obtidos pela aplicação de planejamentos fatoriais de dois níveis. No entanto, os planejamentos usados para o ajuste de modelos quadráticos permitem uma melhor descrição do comportamento dos dados mesmo na presença de curvatura.

Os modelos empíricos são polinômios de segunda ordem, em que a resposta $\hat{y}$ é relacionada às variáveis $x_{i}$ através da seguinte equação: 


$$
\hat{y}=\beta_{0}+\sum_{i=1}^{k} \beta_{i} x_{i}+\sum_{i=1}^{k} \beta_{i i} x_{i}^{2}+\sum_{1 \leq i \leq j}^{k} \beta_{i j} x_{i} x_{j}+\varepsilon
$$

sendo $\mathrm{k}$ o número de variáveis, $\beta_{0}$ o termo constante, $\beta_{\mathrm{i}}, \beta_{\mathrm{ij}}$ e $\beta_{\mathrm{ii}}$ são os parâmetros de regressão para os efeitos dos coeficientes linear, de interação e quadrático, respectivamente e $\varepsilon$ refere-se ao resíduo, isto é, a diferença entre a resposta obtida experimentalmente e a resposta prevista pelo modelo. ${ }^{15}$

\section{Planejamentos experimentais para ajuste de funções quadráticas}

Para a descrição correta de comportamento de dados experimentais que apresentam curvatura, o planejamento experimental usado tem que assegurar que todas as variáveis devem ser estudadas em no mínimo três níveis. Isso é possível com a aplicação de matrizes experimentais que permitem o ajuste de funções de segunda ordem. Os planejamentos experimentais mais usados para o ajuste de funções quadráticas são: Box-Behnken, Composto Central e Doehlert. Os planejamentos fatoriais de três níveis têm limitada aplicação prática, pois, para um número de fatores maior que dois, o número de experimentos para sua execução torna-se muito grande fazendo com que este perca eficiência na modelagem. As matrizes experimentais para aplicação destes planejamentos para duas, três ou mais variáveis podem ser facilmente encontradas em artigos de revisão recentemente publicados ${ }^{2,4,16}$ e por esse motivo não serão apresentadas neste artigo. A Tabela 1 ilustra algumas aplicações destes planejamentos para otimização de métodos analíticos.

\subsection{Planejamento Composto Central}

Este planejamento é formado pela combinação de três partes: (1) um planejamento fatorial de dois níveis fracionário ou completo, (2) pontos axiais, de um planejamento em estrela, no qual os pontos estão a uma distância $\alpha$ do centro e (3) um ponto central. As representações gráficas apresentadas nas figuras 1a e 1b são para otimização de duas e três variáveis respectivamente. Eles foram desenvolvidos por Box e Wilson na década de 1950 como uma alternativa aos planejamentos fatoriais de três níveis que demandavam muitos experimentos para um pequeno número de fatores, mesmo quando se empregavam planejamentos fracionários. Para sua construção, deve-se definir o número de fatores a serem otimizados (k), o tipo de planejamento fatorial $\left(2^{k-p}\right.$, se o fatorial for completo $p=0$, se fracionário $p>0$ e $p$ é um número inteiro) e o número de réplicas do ponto central. O número de experimentos (N) para sua aplicação é dado por $N=k^{2}+2 k$ + pc, onde pc é o número de experimentos no ponto central. Os valores $\alpha$ dos pontos axiais dependem do número de variáveis e podem ser calculados por $\alpha=2^{(k-p) / 4}$. Para duas, três e quatro variáveis, por exemplo, seus valores são, respectivamente, 1,$41 ; 1,68$ e $2,00 .^{17,18}$

\subsection{Planejamento Box-Behnken}

Box e Behnken ${ }^{4}$ sugeriram como selecionar pontos de um planejamento fatorial de três níveis de forma a permitir uma estimativa eficiente e econômica dos coeficientes de primeira e segunda ordem do modelo matemático ajustado. De forma geral, este planejamento requer poucas combinações de fatores sendo estes 
estudados sempre em três níveis $(-1,0$ e +1$)$ e o número de experimentos $(\mathrm{N})$ necessários para sua execução é dado por $\mathrm{N}=\mathrm{k}^{2}+\mathrm{k}+$ pc. $^{4,17}$. No entanto é importante notar que, apesar das vantagens citadas, esse planejamento não testa condições experimentais extremas como, por exemplo, no caso de três variáveis, as combinações de níveis representadas por $(+1,+1,+1)$ e $(-1,-1,-$ 1). Esses pontos representam vértices de um cubo e foram descartados do planejamento $3^{3}$ original para se obter a matrix experimental Box-Behnken. Outra observação se refere ao fato de não se poder obter uma matriz para duas variáveis. Isso ocorre, pois não há como se eliminar pontos de um planejamento $3^{2}$ original garantindo a distribuição homogênea dos pontos pelo espaço experimental e, ao mesmo tempo obter um número suficiente deles para a estimativa dos coeficientes de um modelo quadrático. A representação gráfica para o planejamento usado na otimização de três variáveis é mostrado na Figura 1c.

\subsection{Planejamento Doehlert}

Desenvolvido por David H. Doehlert em 1970, este planejamento vem se mostrando uma alternativa prática e econômica em relação aos outros planejamentos de segunda ordem. O planejamento Doehlert descreve um domínio circular para duas variáveis, esférico para três e hiperesférico para mais de três variáveis, o que acentua a uniformidade no espaço envolvido. As suas matrizes não são rotáveis como nos planejamentos anteriores, mas possui várias vantagens como necessidade de poucos pontos experimentais para sua aplicação e alta eficiência. Embora o planejamento não seja ortogonal, isto não significa perda da qualidade necessária para o seu uso efetivo nos procedimentos de otimização. Outras características deste planejamento são destacadas: (a) O número de experimentos (N) necessários para aplicação do planejamento é dado por $\mathrm{N}=\mathrm{k}^{2}+\mathrm{k}+\mathrm{pc}$, onde $\mathrm{k}$ é o número de variáveis e pc é o número de réplicas do ponto central; (b) Cada variável pode ser estudada em diferentes números de níveis, característica importante quando se tem interesse que alguns fatores sejam estudados em quantidades maiores ou menores de níveis e (c) O deslocamento do planejamento pode ser feito aproveitando-se pontos do planejamento inicial, dando a este planejamento grande mobilidade. ${ }^{4,17,19}$ Para duas variáveis, os pontos experimentais para um planejamento Doehlert é representado pelos vértices de um hexágono mais o ponto central (Figura 1d). Para três variáveis, esse planejamento é representado por um sólido geométrico chamado de cubooctaedro que, a depender de como é projetado no plano, pode gerar matrizes experimentais diferentes. A Figura 1e mostra três projeções planas que geram diferentes matrizes experimentais na otimização de três variáveis.

\section{Ajuste de modelo matemático}

Após realização dos experimentos e obtenção das respostas relacionadas a cada ponto experimental ajusta-se uma função matemática com o objetivo de descrever o comportamento das respostas de acordo com a variação dos níveis das variáveis estudadas. Em outras palavras, devem-se estimar os coeficientes $b$ da equação 4 que em notação matricial, pode ser representada por:

$$
[y]=[X][b] \quad \text { Eq. } 4
$$

Na qual $[y]$ é o vetor resposta, $[X]$ é a matriz experimental do planejamento escolhido e [b] é o vetor constituído pelos coeficientes do modelo ajustado. A equação 4 pode ser resolvida usando-se o método dos mínimos quadrados, o qual é uma técnica de regressão múltipla usada no ajuste de uma função matemática aos dados experimentais 
Tabela 1. Algumas aplicações recentes da metodologia de superfícies de resposta para otimização de métodos analíticos

\begin{tabular}{|c|c|c|c|c|c|c|}
\hline Analitos & Amostras & Sistema analítico & Tipo de planejamento & Fatores otimizados & Respostas & Ref. \\
\hline $\mathrm{Cu}, \mathrm{Fe}, \mathrm{Mn}, \mathrm{Ni}$ & Urina & FIA-FAAS & $\begin{array}{l}\text { Composto central } \\
\text { ortogonal }\end{array}$ & $\begin{array}{l}\text { Concentração do tampão acetato } \\
\text { de amônio, vazão de fluxo da } \\
\text { amostra, volume e concentração } \\
\text { do eluente }\end{array}$ & $\begin{array}{l}\text { Percentual de } \\
\text { recuperação }\end{array}$ & 20 \\
\hline $\mathrm{Cu}$ & $\begin{array}{c}\text { Material } \\
\text { particulado }\end{array}$ & $\begin{array}{c}\text { Amostragem em } \\
\text { suspensão/CVG AAS }\end{array}$ & Box-Behnken & $\begin{array}{l}\text { Concentrações de } \mathrm{HCl}, \mathrm{NaBH}_{4} \text { e } \\
\text { sulfanilamida, volume de } \mathrm{NaBH}_{4}\end{array}$ & Absorbância & 21 \\
\hline $\mathrm{Cu}$ & Água e peixe & $\begin{array}{c}\text { Polímeros impressos com } \\
\text { íons/FAAS }\end{array}$ & Box-Behnken & $\begin{array}{c}\text { pH da solução de adsorção, } \\
\text { quantidade do polímero, tempo } \\
\text { de adsorção }\end{array}$ & $\begin{array}{l}\text { Percentual de } \\
\text { adsorção }\end{array}$ & 22 \\
\hline $\mathrm{Se}$ & $\begin{array}{c}\text { Plasma } \\
\text { sanguíneo }\end{array}$ & ETAAS & Doehlert & $\begin{array}{l}\text { Temperaturas de pirólise e } \\
\text { atomização, tempo de pirólise }\end{array}$ & $\begin{array}{l}\text { Absorbância e } \\
\text { ruído }\end{array}$ & 23 \\
\hline $\mathrm{Cd}$ & Óleos vegetais & $\begin{array}{c}\text { Microextração de gota } \\
\text { única assistida por micro- } \\
\text { ondas/ } \\
\text { HR-CS-ETAAS }\end{array}$ & Box-Behnken & $\begin{array}{l}\text { Amplitude de sonicação, tempo e } \\
\text { temperatura da extração }\end{array}$ & Absorbância & 24 \\
\hline $\mathrm{Sb}, \mathrm{Bi}, \mathrm{Sn}, \mathrm{Hg}$ & $\begin{array}{l}\text { Água de rio e de } \\
\text { mar }\end{array}$ & FIA-SPE-CVG-HR-CS-ETAAS & $\begin{array}{l}\text { Composto central } \\
\text { rotacional }\end{array}$ & $\begin{array}{c}\text { Vazão de fluxo do eluente e da } \\
\text { amostra, concentração do eluente } \\
\text { e do agente redutor }\end{array}$ & Razão sinal/ruído & 25 \\
\hline $\mathrm{Sb}$ & $\begin{array}{l}\text { Águas naturais e } \\
\text { medicamentos } \\
\text { anti- } \\
\text { leishmaniose }\end{array}$ & $\begin{array}{l}\text { Extração em ponto nuvem } \\
\text { em } \\
\text { fluxo/Espectrofotometria }\end{array}$ & Box-Behnken & $\begin{array}{l}\text { Concentrações de ácido ascórbico, } \\
\qquad \mathrm{KI}, \mathrm{H}_{2} \mathrm{SO}_{4} \text {, e TritonX-114 }\end{array}$ & Absorbância & 26 \\
\hline Sulfatiazol & Leite & Fluorescência molecular & D-ótimo & $\begin{array}{c}\text { Tipo de leite, razão entre o } \\
\text { volume de ácido tricloroacético e } \\
\text { leite, velocidade e temperatura de } \\
\text { centrifugação, tempo de } \\
\text { derivatização, volume de } \\
\text { fluoresceína }\end{array}$ & $\begin{array}{l}\text { Percentual de } \\
\text { recuperação }\end{array}$ & 27 \\
\hline
\end{tabular}




\begin{tabular}{|c|c|c|c|c|c|c|}
\hline $\mathrm{Sb}(\mathrm{III}), \mathrm{Sb}(\mathrm{V}), \mathrm{TMSb}$ & Águas naturais & $\begin{array}{c}\text { MSFIA/HG/Fluorescência } \\
\text { atômica }\end{array}$ & Box-Behnken & Concentrações de $\mathrm{HCl}, \mathrm{NaBH}_{4}$ e KI & $\begin{array}{l}\text { Intensidades de } \\
\text { fluorescência } \\
\text { convertidas em } \\
\text { desejabilidade }(D)\end{array}$ & 28 \\
\hline Ésteres de ftalatos & $\begin{array}{l}\text { Cosméticos e } \\
\text { águas naturais }\end{array}$ & $\begin{array}{l}\text { Microextração em gota } \\
\text { orgânica solidificada por } \\
\text { emulsão assistida por } \\
\text { ultrassom/HPLC }\end{array}$ & Box-Behnken & $\begin{array}{l}\text { Volume do solvente de extração, } \\
\text { força iônica, tempo de extração, } \\
\text { tempo de centrifugação }\end{array}$ & Área de pico & 29 \\
\hline Carboidratos & Alimentos & $\begin{array}{c}\text { Cromatografia } \\
\text { Eletrocinética Micelar }\end{array}$ & Composto central & $\begin{array}{l}\mathrm{pH} \text {, concentrações do eletrólito e } \\
\text { surfactante }\end{array}$ & $\begin{array}{l}\text { Resolução entre } \\
\text { picos adjacente }\end{array}$ & 30 \\
\hline $\begin{array}{l}\text { Estreptomicina e } \\
\text { dihidroestreptomicina }\end{array}$ & $\begin{array}{c}\text { Dosagens } \\
\text { farmacêuticas } \\
\text { veterinárias }\end{array}$ & $\begin{array}{c}\text { HPLC/Detecção } \\
\text { amperométrica pulsada }\end{array}$ & Composto central & $\begin{array}{l}\text { Potenciais (determinação, } \\
\text { ativação, limpeza) }\end{array}$ & Área de pico & 31 \\
\hline $\begin{array}{l}\text { Compostos orgânicos } \\
\text { voláteis }\end{array}$ & Manga & HS-SPME/CG-MS & Doehlert & $\begin{array}{c}\text { Temperatura de extração e } \\
\text { dessorção, tempo de extração e } \\
\text { dessorção }\end{array}$ & Área de pico & 32 \\
\hline $\begin{array}{l}\text { 2,4,6-tricloroanisol, } \\
\text { 2,4,6-tribromoanisol }\end{array}$ & Vinho branco & HS-SDME/CG-ECD & Box-Behnken & $\begin{array}{l}\text { Tempo e temperatura de } \\
\text { extração, pH, força iônica, tipo de } \\
\text { amostra }\end{array}$ & $\begin{array}{l}\text { Média geométrica } \\
\text { entre as áreas de } \\
\text { pico }\end{array}$ & 33 \\
\hline $\begin{array}{c}\text { Pesticidas } \\
\text { organoclorados }\end{array}$ & Leite bovino & HS-SDME/CG-ECD & Doehlert & Tempo e temperatura de extração & Área de pico & 34 \\
\hline $\begin{array}{l}\mathrm{Al}, \mathrm{Ca}, \mathrm{Cd}, \mathrm{Cr}, \mathrm{Cu}, \mathrm{Ba} \\
\mathrm{Fe}, \mathrm{K}, \mathrm{Mg}, \mathrm{Mn}, \mathrm{P}, \mathrm{S}, \mathrm{Sr} \\
\mathrm{Zn}\end{array}$ & $\begin{array}{l}\text { Ração para } \\
\text { cachorro e gato }\end{array}$ & ICP OES & Fatorial de 3 níveis & $\begin{array}{l}\text { Potência de radiofrequência, } \\
\text { vazão de fluxo de nebulização }\end{array}$ & $\begin{array}{c}\mathrm{Mg} \mathrm{II/Mg} \mathrm{I} \\
\text { convertida em } \\
\text { resposta múltipla }\end{array}$ & 35 \\
\hline Se & $\begin{array}{l}\text { Bebidas } \\
\text { argentinas }\end{array}$ & SPE/FIA-HG-ICP OES & Composto central & $\begin{array}{l}\text { Vazões de fluxo da amostra, do } \\
\text { ácido e do reagente, concentração } \\
\text { do ácido }\end{array}$ & $\begin{array}{l}\text { Intensidade de } \\
\text { emissão }\end{array}$ & 36 \\
\hline $\mathrm{Cd}, \mathrm{Cu}, \mathrm{Fe}, \mathrm{Pb}, \mathrm{Zn}$ & Gasolina e diesel & HF-SPME/ICP OES & Composto central & $\begin{array}{l}\text { pH da amostra, concentração do } \\
\text { eluente, quantidade de fase } \\
\text { receptora, tempo de extração }\end{array}$ & $\begin{array}{l}\text { Percentual de } \\
\text { recuperação }\end{array}$ & 37 \\
\hline
\end{tabular}




\begin{tabular}{|c|c|c|c|c|c|c|}
\hline $\mathrm{U}(\mathrm{VI})$ & Água & ELL/ICP-MS & Doehlert & $\begin{array}{l}\text { Concentrações de ácido fosfórico, } \\
\text { cítrico e bis(2-etilexil)fosfórico }\end{array}$ & $\begin{array}{l}\text { Percentual de } \\
\text { recuperação }\end{array}$ & 38 \\
\hline As, Bi, Ge, Hg, Sb, Sn & $\begin{array}{l}\text { Material de } \\
\text { referência } \\
\text { certificado }\end{array}$ & MSIS/ICP OES & Composto central & $\begin{array}{l}\text { Vazões de fluxo da amostra e do } \\
\text { aerossol, altura de observação, } \\
\text { concentrações de } \mathrm{NaBH}_{4} \text { e } \mathrm{HNO}_{3}\end{array}$ & Mg II/Mg I & 39 \\
\hline $\mathrm{V}, \mathrm{Cr}, \mathrm{Fe}, \mathrm{Co}, \mathrm{Ni}, \mathrm{As}, \mathrm{Se}$ & $\begin{array}{c}\text { Gêneros } \\
\text { alimentícios }\end{array}$ & ICP-MS & Composto central & $\begin{array}{c}\text { Fluxos de } \mathrm{He} \mathrm{e} \mathrm{H}_{2} \text {, deriva do } \\
\text { hexapolo e do quadrupolo }\end{array}$ & Razão sinal/ruído & 40 \\
\hline Se, As & $\begin{array}{l}\text { Sedimentos } \\
\text { estuarinos }\end{array}$ & HG ICP OES & Doehlert & $\begin{array}{c}\text { Vazões de fluxo da amostra, } \\
\mathrm{NaBH}_{4} \text { e } \mathrm{HCl} \text {, pressão do } \\
\text { nebulizador }\end{array}$ & $\begin{array}{c}\text { Intensidades de } \\
\text { emissão } \\
\text { convertidas em } \\
\text { Resposta Múltipla }\end{array}$ & 41 \\
\hline $\mathrm{Ag}, \mathrm{Al}, \mathrm{As}, \mathrm{Mn}, \mathrm{Ti}$ & Gasolina e diesel & HF-LPME/ICP OES & Box-Behnken & $\begin{array}{c}\mathrm{pH}, \text { tempo de extração, } \\
\text { concentrações de APDC e } \mathrm{HNO}_{3}\end{array}$ & $\begin{array}{l}\text { Percentual de } \\
\text { recuperação }\end{array}$ & 42 \\
\hline $\mathrm{Pb}$ & Água de rio & $\begin{array}{c}\text { Co-precipitação assistida } \\
\text { por ultrassom/ICP OES }\end{array}$ & Doehlert & $\begin{array}{c}\text { Tempo de sonicação, } \\
\text { concentrações de } \mathrm{KMnO}_{4} \text { e } \mathrm{MnSO}_{4}\end{array}$ & $\begin{array}{l}\text { Percentual de } \\
\text { recuperação }\end{array}$ & 43 \\
\hline $\begin{array}{c}\text { Hidroquinona, catecol, } \\
\text { acetaminofeno, 4- } \\
\text { nitrofenol }\end{array}$ & Águas residuais & Voltametria cíclica & Doehlert & $\begin{array}{l}\text { Amplitude do pulso, frequência, } \\
\text { etapa de potencial }\end{array}$ & $\begin{array}{c}\text { Corrente } \\
\text { convertida em } \\
\text { desejabilidade }(D) \\
\end{array}$ & 44 \\
\hline $\mathrm{Zn}, \mathrm{Cu}, \mathrm{Pb}, \mathrm{Cd}$ & Gasolina & $\begin{array}{l}\text { Digestão assistida por } \\
\text { micro-ondas/Voltametria de } \\
\text { redissolução anódica }\end{array}$ & Fatorial completo $2^{2}$ & pH, potencial de eletrodeposição & Corrente & 45 \\
\hline Aflotoxinas B1 e B2 & Amendoim & $\begin{array}{l}\text { Voltametria adsortiva com } \\
\text { redissolução catódica }\end{array}$ & Fatorial completo $2^{2}$ & $\mathrm{pH}$, potencial de acumulação & Corrente de pico & 46 \\
\hline $\mathrm{Cr}(\mathrm{III}), \mathrm{Cr}(\mathrm{VI})$ & $\begin{array}{l}\text { Amostras } \\
\text { sintéticas }\end{array}$ & $\begin{array}{c}\text { Voltametria de onda } \\
\text { quadrada }\end{array}$ & Composto central & pH, concentração de DTPA & $\begin{array}{l}\text { Redução da altura } \\
\text { de pico }\end{array}$ & 47 \\
\hline $\begin{array}{l}\text { Melatonina e seus } \\
\text { metabólitos }\end{array}$ & $\begin{array}{c}\text { Material } \\
\text { certificado }\end{array}$ & $\begin{array}{c}\text { Eletroforese capilar/HPLC- } \\
\text { DAD }\end{array}$ & Fatorial completo $2^{4}$ & $\begin{array}{c}\text { Concentrações de metanol e } \\
\text { acetato de sódio, vazão de fluxo, } \\
\mathrm{pH}\end{array}$ & $\begin{array}{l}\text { Resolução, tempo } \\
\text { de migração, pico }\end{array}$ & 48 \\
\hline Compostos fenólicos & Óleo de oliva & Eletroforese capilar/HPLC & Composto central & $\begin{array}{l}\mathrm{pH} \text {, voltagem, concentração de } \\
\text { ácido bórico }\end{array}$ & $\begin{array}{c}\text { Resolução e } \\
\text { tempo de corrida }\end{array}$ & 49 \\
\hline
\end{tabular}




\begin{tabular}{|c|c|c|c|c|c|c|}
\hline & & & & & $\begin{array}{c}\text { convertida em } \\
\text { desejabilidade }(D)\end{array}$ & \\
\hline Ácidos fenólicos & Frutas exóticas & Eletroforese capilar/ELL & Fatorial $3^{2}$ & $\begin{array}{c}\text { Concentrações de tetraborato de } \\
\text { sódio e metanol }\end{array}$ & $\begin{array}{l}\text { Resolução entre } \\
\text { picos convertida } \\
\text { em desejabilidade } \\
(D)\end{array}$ & 50 \\
\hline $\mathrm{Cu}$ & $\begin{array}{l}\text { Folhas vegetais e } \\
\text { água de rio }\end{array}$ & $\begin{array}{l}\text { Espectrofotometria em fase } \\
\text { sólida }\end{array}$ & Doehlert & Complexante e surfactante & Absorbância & 51 \\
\hline $\mathrm{Zn}$ & $\begin{array}{c}\text { Águas e folhas } \\
\text { vegetais }\end{array}$ & $\begin{array}{c}\text { Extração usando minicoluna } \\
\text { com polímero impresso } \\
\text { ionicamente e } \\
\text { determinação por FAAS }\end{array}$ & Doehlert & $\begin{array}{l}\mathrm{pH} \text {, concentração do tampão e } \\
\text { vazão de fluxo }\end{array}$ & Absorbância & 52 \\
\hline Amitriptilina & $\begin{array}{l}\text { Formulações } \\
\text { farmacêuticas }\end{array}$ & FIA/turbidimetria & Doehlert & $\begin{array}{c}\mathrm{pH}, \text { vazão do carreador, } \\
\text { concentração do surfactante e } \\
\text { comprimento da alça de } \\
\text { amostragem }\end{array}$ & Turbidez $(-\log T)$ & 53 \\
\hline $\mathrm{Ag}(\mathrm{I})$ & $\begin{array}{l}\text { Águas de } \\
\text { represa }\end{array}$ & $\begin{array}{l}\text { Voltametria de redissolução } \\
\text { anódica empregando } \\
\text { eletrodo de pasta de } \\
\text { carbono }\end{array}$ & Composto central & $\begin{array}{l}\text { Tempo de pré-concentração e } \\
\text { velocidade de varredura }\end{array}$ & Corrente & 54 \\
\hline Desoxinivalenol & - & Cromatografia gasosa & Composto central & $\begin{array}{c}\text { Quantidade de TFAA e } \mathrm{NaHCO}_{3} \\
\text { tempo e temperatura }\end{array}$ & $\begin{array}{l}\text { Área do pico } \\
\text { cromatográfico }\end{array}$ & 55 \\
\hline Bifenilas policloradas & Leite materno & $\begin{array}{c}\text { HS-SPME/Cromatografia } \\
\text { gasosa }\end{array}$ & Doehlert & $\begin{array}{l}\text { Concentração do } \mathrm{NaCl} \text {, } \\
\text { temperatura, tempo e } \\
\text { concentração de metanol }\end{array}$ & $\begin{array}{l}\text { Área do pico } \\
\text { cromatográfico }\end{array}$ & 56 \\
\hline
\end{tabular}

APDC - Pirrolidina Ditiocarbamato de Amônio; CVG - Geração de Vapor Químico; CG - Cromatografia Gasosa; DAD - Detecção por Arranjo de Diodos; DTPA - Ácido Dietilenotriaminopentacético; ECD - Detecção por Captura de Elétrons; ELL - Extração Líquido-Líquido; ETAAS - Espectrometria de Absorção Atômica com Atomização Eletrotérmica; FAAS Espectrometria de Absorção Atômica com Chama; FIA - Análise com Injeção em Fluxo; HF-LPME - Microextração em fase líquida com fibra oca; HF- SPME - Microextração em fase sólida com fibra oca; HG - Geração de Hidretos; HPLC - Cromatografia Líquida de Alta Eficiência; HR-CS - Fonte Contínua de Alta Resolução; HS-SDME - Microextração em headspace com única gota; HSSPME- Microextração em fase sólida em modo headspace; ICP-MS - Espectrometria de Massas com Plasma Acoplado Indutivamente; ICP OES - Espectrometria de Emissão Óptica com Plasma Acoplado Indutivamente; MSFIA - Análise com Injeção em Fluxo Multi-Seringa; MSIS - Sistema de Introdução de Amostras Multi-Modo; PAD - Detector Amperométrico Pulsado; RP-UPLC Cromatografia Líquida de Ultra Eficiência em Fase Reversa; SIA - Análise com Injeção Sequencial; SPE - Extração em Fase Sólida; SPME - Micro-extração em Fase Sólida; UV - Ultravioleta. 
(a)

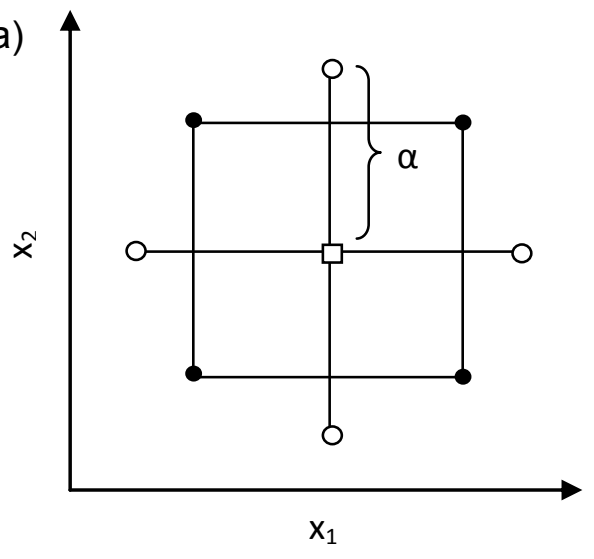

(c)

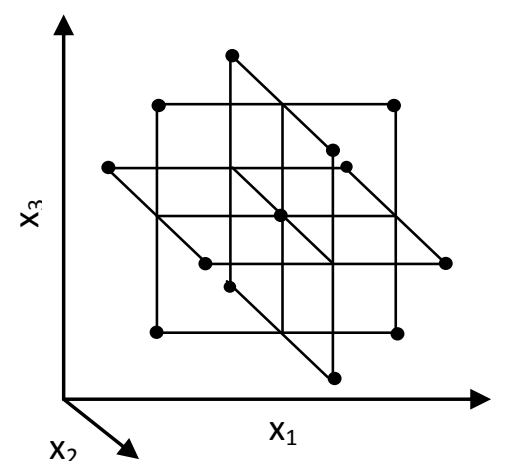

(b)

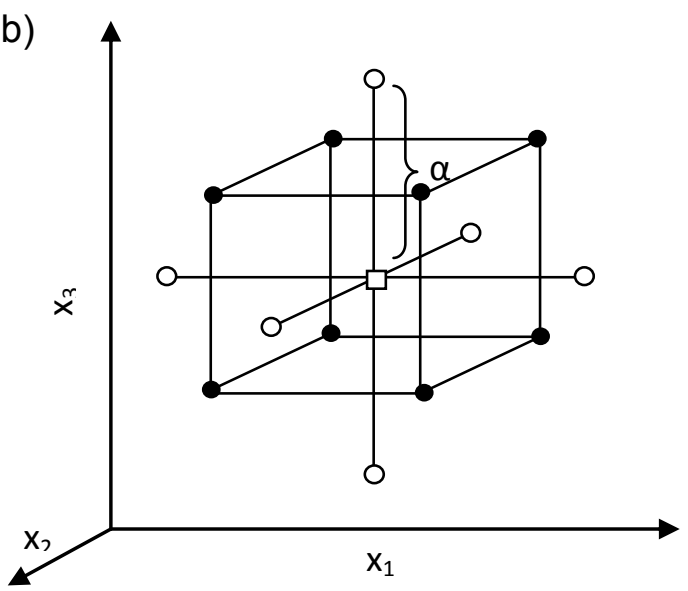

(d)

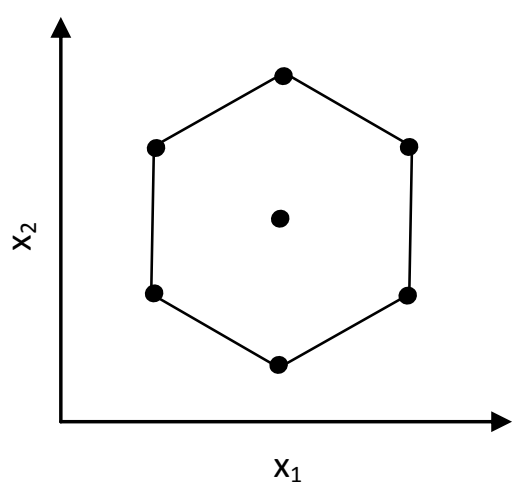

(e)
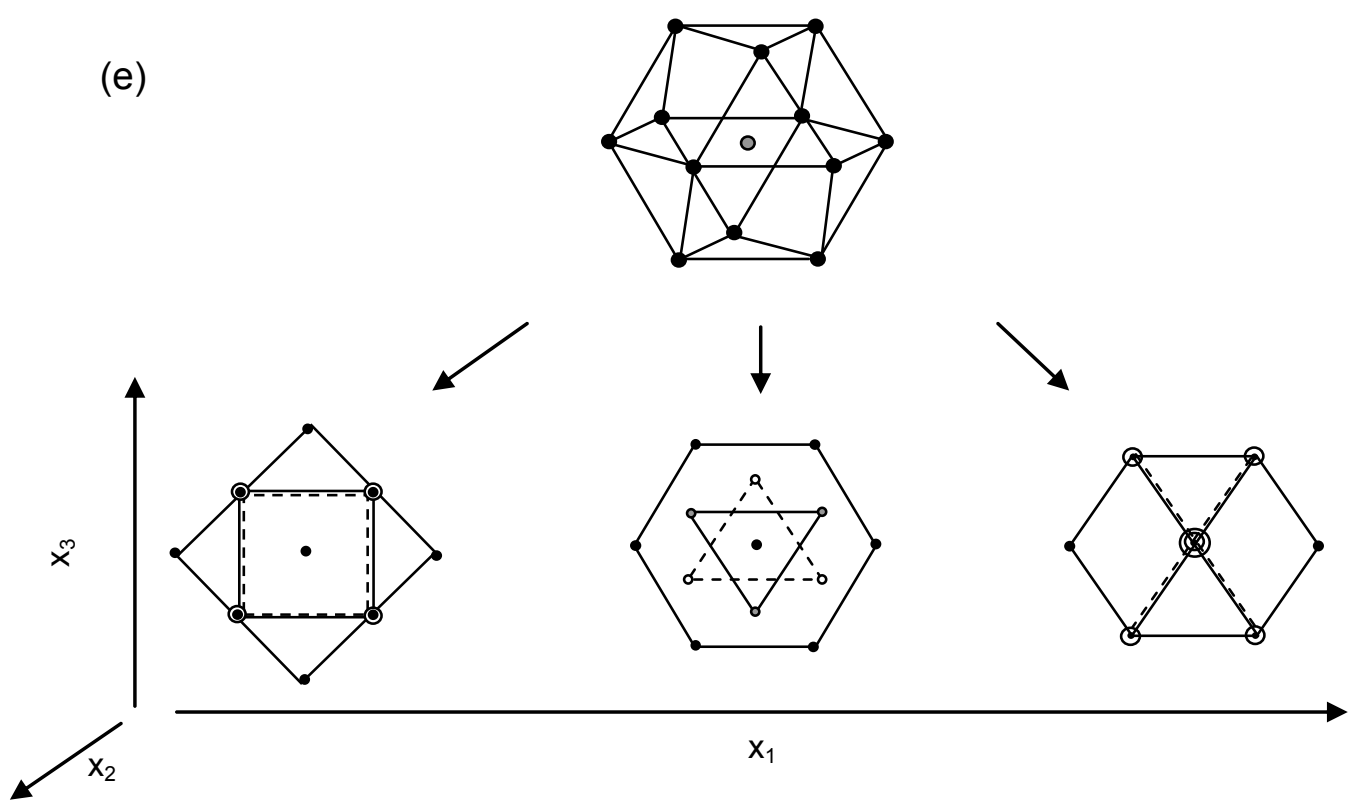

Figura 1. Pontos experimentais para geração de planejamentos para ajuste de modelos quadráticos: (a) planejamento composto central para duas variáveis, (b) planejamento composto central para três variáveis, (c) planejamento Box-Benhken para três variáveis, (d) planejamento Doehlert para duas variáveis e (e) três planejamentos Doehlert para otimização de três variáveis gerados pela projeção do sólido geométrico cubooctraedro no plano

de forma a gerar os menores resíduos possíveis. A equação 4 pode ser trabalhada, 
levando-se em consideração as propriedades das matrizes, para originar a seguinte equação:

$$
[b]=\left([X]^{T}[X]\right)^{-1}\left([X]^{T}[y]\right) \quad \text { Eq. } 5
$$

A grande vantagem da aplicação da equação 5 é a baixa demanda de processamento computacional necessário para a determinação dos coeficientes do vetor $b$. A equação encontrada descreve a superfície de resposta e, desta forma, o comportamento dos dados experimentais obtidos. $^{6,57,58}$

No método dos mínimos quadrados assume-se que os erros apresentam um perfil de distribuição randômica com média zero e variância desconhecida comum e que esses erros são independentes uns dos outros. Desta forma, a variância estimada para cada componente do vetor [b] é comumente obtida por repetições autênticas do ponto central de acordo com a equação 6 :

$$
[\hat{V}(b)]=\left([X]^{T}[X]\right)^{-1} s^{2} \quad \text { Eq. } 6
$$

Então, a extração da raiz quadrada para cada componente de $\widehat{V}$ permite a obtenção dos erros padrão para os coeficientes $b$ que compõem a equação da superfície de resposta, permitindo a avaliação de sua significância. ${ }^{6}$

\subsection{Avaliação do modelo matemático ajustado}

A avaliação do modelo matemático ajustado deve ser realizada para certificar que ele é adequado na descrição do comportamento dos dados experimentais. Uma forma de realizar esta avaliação é através da Analise de variância (ANOVA), comparando-se a variação devido ao tratamento (mudança na combinação dos níveis das variáveis) com a flutuação aleatória das respostas obtidas (erros randômicos). Através desta comparação, torna-se possível avaliar a significância da regressão usada e a qualidade de seu ajuste às respostas. ${ }^{6,16,53}$

Na ANOVA, a variância total dos dados é dividida em duas contribuições principais: a soma quadrática explicada pela regressão $\left(\mathrm{SQ}_{\mathrm{R}}\right)$ e a soma quadrática residual $\left(\mathrm{SQ}_{\mathrm{r}}\right)$. Ambas somas são tomadas para todos os níveis do planejamento experimental, $\mathrm{i}=$ $1,2, \ldots, \mathrm{m}$ e para todas as réplicas realizadas em cada nível $\mathrm{j}=1,2, \ldots, \mathrm{n}_{\mathrm{i}}$. $\mathrm{SQ}_{\mathrm{R}}$ é a soma dos quadrados das diferenças entre os valores preditos pela regressão e a média de todos os valores de respostas e apresenta $p-1$ graus de liberdade, onde $p$ é o número de coeficientes do modelo. $\mathrm{SQ}_{\mathrm{r}}$ é a soma dos quadrados das diferenças (ou resíduos) entre todos os valores experimentais e os valores preditos do modelo. Ele possui $n-p$ graus de liberdade, onde $\mathrm{n}$ é o número total de dados experimentais usados para determinar o modelo. Altos valores de $\mathrm{SQ}_{R}$ e pequenos valores de $\mathrm{SQ}_{\mathrm{r}}$ são indicativos de que os modelos descrevem com exatidão os dados experimentais obtidos. A soma desses dois termos é igual à soma quadrática total $\left(\mathrm{SQ}_{\mathrm{T}}\right)$, que é a soma dos quadrados das diferenças entre os valores experimentais e a média do conjunto dos dados. Esta soma possui $n-1$ graus de liberdade e representa a variância total nos dados.

Se houver replicação de algum(ns) ponto(s) experimental(is), a soma quadrática residual $\left(\mathrm{SQ}_{\mathrm{r}}\right)$ ainda pode ser decomposta em duas contribuições: a soma quadrática da falta de ajuste $\left(\mathrm{SQ}_{\mathrm{faj}}\right)$ e do erro puro $\left(\mathrm{SQ}_{\mathrm{ep}}\right)$. $\mathrm{A}$ $\mathrm{SQ}_{\mathrm{faj}}$ é a soma dos quadrados das diferenças entre os valores preditos a cada nível e o valor experimental médio em cada nível e possui $m-p$ graus de liberdade. $A S_{e p}$ é a soma dos quadrados das diferenças entre todos os valores experimentais e a média dos valores experimentais no mesmo nível. Esta quantidade possui $n-m$ graus de liberdade onde $\mathrm{m}$ é o número de níveis distintos do planejamento experimental. ${ }^{5,6,53}$ 
Apenas a avaliação das somas quadráticas poderia levar ao erro de considerar somente a contribuição do polinômio ajustado na qualidade do modelo. Com este raciocínio, quanto maior for a $S_{R}$ melhor seria o modelo e, consequentemente, o melhor modelo seria aquele que tivesse a equação mais longa. No entanto, cada termo acrescentado tira do modelo um grau de liberdade, pois o número de parâmetros estatísticos a serem avaliados diminui em relação às determinações independentes. Como os números de graus de liberdade (gl) devem ser levados em conta nos argumentos estatísticos, para avaliar se o termo adicionado à função matemática compensa o grau de liberdade perdido e contribui na melhor explicação da variação dos dados se adota um novo termo chamado de média quadrática (MQ). As $\mathrm{MQ}_{\mathrm{s}}$ são calculadas dividindo-se as $\mathrm{SQ}_{s}$ de cada fonte de variação pelos seus respectivos graus de liberdade. ${ }^{59}$ As equações que relacionam as fontes de variação para o cálculo das $\mathrm{SQ}_{s}$ e $\mathrm{MQ}_{\mathrm{s}}$ são apresentadas na Tabela 2.

A significância estatística da regressão pode ser avaliada usando-se a razão entre as médias quadráticas da regressão $\left(\mathrm{MQ}_{\text {reg }}\right)$ e a média quadrática dos resíduos $\left(\mathrm{MQ}_{\text {res }}\right)$ e por comparação do resultado com o valor apropriado proveniente da distribuição de Fisher, levando-se em consideração seus respectivos graus de liberdade associada às variâncias da regressão $\left(\mathrm{v}_{\mathrm{reg}}\right)$ e dos resíduos $\left(v_{\text {res }}\right)$ :

$$
\frac{M Q_{r e g}}{M Q_{r e s}} \approx F_{v_{r e g}, v_{r e s}}
$$

Eq. 7

Então, um valor estatisticamente significante para esta razão deve ser maior que o valor de $F$ tabelado. Isto é uma indicação de que o modelo matemático está bem ajustado aos dados experimentais.
Outra forma de avaliar a qualidade do modelo é o teste de falta de ajuste. Se o modelo matemático está bem ajustado aos dados experimentais, $\mathrm{MQ}_{\mathrm{faj}}$ deve, como a $M Q_{e p}$ refletir apenas os erros randômicos inerentes ao sistema investigado. É possível utilizar a distribuição $F$ para avaliar se há alguma diferença significativa entre estas duas médias da mesma forma que foi feito para a avaliação da significância estatística da regressão:

$$
\frac{M Q_{f a j}}{M Q_{e p}} \approx F_{v_{f_{a j}, v_{e p}}}
$$

Onde $v_{\text {faj }}$ e $v_{\text {ep }}$ são, respectivamente, os graus de liberdade associados com as variâncias da falta de ajuste e do erro puro. Se esta razão for maior que o valor de $\mathrm{F}$ tabelado há evidência de falta de ajuste e o modelo deve ser melhorado. Contudo, se este valor é menor que o valor de $\mathrm{F}$ tabelado, o modelo ajustado pode ser considerado satisfatório. Para aplicar o teste de falta de ajuste, os experimentos determinados pela matriz experimental devem ser realizados com repetições autênticas ao menos em seu ponto central.

Em resumo, para um modelo ser considerado bem ajustado aos dados experimentais ele deve apresentar uma regressão significativa e uma falta de ajuste não significativa. A maior parte da variação total das observações em torno da média deve ser descrita pela equação de regressão e a variação e o restante ficarão com os resíduos. Além disso, a maior parte da variação relacionada com os resíduos deve ser atribuída ao erro puro e não à falta de ajuste, a qual é diretamente atribuída à qualidade do modelo. ${ }^{5,6,60,61}$

Tabela 2. Análise de variância para avaliação do modelo matemático ajustado aos dados experimentais usando regressão múltipla 


\begin{tabular}{|l|c|c|c|}
\hline Fonte de variação & SQ & g.l. & MQ \\
\hline Regressão & $S Q_{\text {reg }}=\sum_{i}^{m} \sum_{j}^{n_{i}}\left(\hat{y}_{i}-\bar{y}\right)^{2}$ & $\mathrm{p}-1$ & $M Q_{\text {reg }}=\frac{S Q_{\text {reg }}}{p-1}$ \\
\hline Resíduos & $S Q_{\text {res }}=\sum_{i}^{m} \sum_{j}^{n_{i}}\left(y_{i j}-\hat{y}_{i}\right)^{2}$ & $\mathrm{n}-\mathrm{p}$ & $M Q_{\text {res }}=\frac{S Q_{\text {res }}}{n-p}$ \\
\hline Falta de ajuste & $S Q f_{f a j}=\sum_{i}^{m} \sum_{j}^{n_{i}}\left(\hat{y}_{i}-\bar{y}_{i}\right)^{2}$ & $\mathrm{~m}-\mathrm{p}$ & $M Q_{f a j}=\frac{S Q_{f a j}}{m-p}$ \\
\hline Erro puro & $S Q_{p e}=\sum_{i}^{m} \sum_{j}^{n_{i}}\left(y_{i j}-\bar{y}_{i}\right)^{2}$ & $\mathrm{n}-\mathrm{m}$ & $M Q_{p e}=\frac{S Q_{p e}}{n-m}$ \\
\hline Total & $S Q_{t o t}=\sum_{i}^{m} \sum_{j}^{n_{i}}\left(y_{i j}-\bar{y}\right)^{2}$ & $\mathrm{n}-1$ & \\
\hline
\end{tabular}

$\mathrm{SQ}$, soma quadrática; $\mathrm{MQ}$, média quadrática; g.I., grau de liberdade; $\mathrm{n}_{\mathrm{i}}$, número de observações; $m$, número total de níveis no planejamento; $p$, número de parâmetros do modelo; $\hat{y}_{i}$, valor estimado pelo modelo para o nível i; $\bar{y}$, média global; $y_{i j}$, réplica realizada em cada nível individual; $\bar{y}_{i}$, média das réplicas realizadas no mesmo conjunto de condições experimentais.

Uma hipótese nula $\left(H_{0}\right)$ também pode ser formulada considerando-se que não há diferença entre as fontes de variação comparadas. Duas possibilidades podem ser consideradas no estabelecimento da seguinte regra de decisão:

a) Rejeição da $\mathrm{H}_{0}$ com uma significância definida pelo pesquisador, quando o valor calculado de $F\left(F_{\text {calc }}\right)$ for localizado fora do intervalo delimitado por $F_{\alpha}\left(\left|F_{\text {calc }}\right| \geq F_{\alpha}\right)$, ou seja, $\boldsymbol{p} \leq \boldsymbol{\alpha}$. Isso significa que o valor observado é significativo no nível de confiança estabelecido (geralmente usa-se $95 \%$ ) e as fontes de variação comparadas são diferentes;

b) Aceitação da $\mathrm{H}_{0}$ que é o caso contrário do que foi descrito em (a).

Um exemplo. Se $\mathrm{H}_{0}$ for rejeitada para a razão $M Q_{\mathrm{faj}} / \mathrm{MQ}_{\mathrm{ep}}$, ter-se-á $\mathrm{p} \leq \alpha$

$$
R^{2}=\frac{S Q_{r e g}}{S Q_{t o t}}
$$

Quanto mais próximo de 1 o valor do coeficiente estiver, melhor será o ajuste do modelo às respostas observadas. Embora este coeficiente seja uma medida de como o ajuste do modelo se aproxima dos dados experimentais, ele não pode ser usado para (frequentemente usa-se $\alpha=0,05$ ), então pode-se dizer que a falta de ajuste para 0 modelo usado é significativa neste nível de probabilidade e, portanto, este modelo não é adequado para a descrição dos dados. ${ }^{62}$

Outros parâmetros que podem ser usados para observar se toda a variação em torno da média está sendo explicada pela regressão são: a percentagem de variação explicada e o gráfico de resíduos.

A percentagem de variação explicada é representada como $R^{2}$, o coeficiente de determinação que varia de 0 a 1 . $O$ valor de $R^{2}$ representa a fração da variação que é explicada pela regressão ou pelos coeficientes do modelo e pode ser calculada pela seguinte equação:

Eq. 9

julgar a sua falta de ajuste porque ele não leva em consideração o número de graus de liberdade para a determinação do modelo.

Levando-se em consideração que o erro puro não explica nada do modelo, deve-se usar um outro parâmetro para descrever a 
contribuição da função ajustada. Este parâmetro é a percentagem máxima de variação explicável que é dada pela seguinte expressão:

$$
R^{2} \text { máxima }=\frac{S Q_{t o t}-S Q_{e p}}{S Q_{t o t}} \times 100 \% \quad \text { Eq. } 10
$$

A análise visual dos gráficos de resíduos pode também gerar valiosas informações sobre a adequação do modelo. Se o modelo matemático estiver bem ajustado, seu gráfico de resíduos apresentará um comportamento que irá sugerir uma distribuição normal dos dados. Se um modelo gerar resíduos de grande magnitude, ele não estará sendo adequado para fazer inferências precisas sobre o comportamento dos dados no campo experimental em estudo. Contudo, se o modelo necessitar de algum outro termo, o gráfico de resíduo apresentará um comportamento que irá indicar que tipo de termo deverá ser adicionado ao modelo. ${ }^{6}$

\section{Determinação das condições} ótimas

A determinação das condições ótimas para os dados modelados pode ser realizada a partir da equação da regressão obtida ou,

$$
\begin{aligned}
& y=b_{0}+b_{1} x_{1}+b_{2} x_{2}+b_{11} x_{1}^{2}+b_{22} x_{2}^{2}+b_{12} x_{1} x_{2} \\
& \frac{\partial y}{\partial x_{1}}=b_{1}+2 b_{11} x_{1}+b_{12} x_{2}=0 \\
& \frac{\partial y}{\partial x_{2}}=b_{2}+2 b_{22} x_{2}+b_{12} x_{1}=0
\end{aligned}
$$

Desta forma, para calcular as coordenadas do ponto crítico, é necessário resolver o sistema do primeiro grau formado pelas equações 12 e 13 e calcular os valores para $x_{1}$ e $x_{2}$. de uma forma mais rudimentar, por análise visual das superfícies de respostas. A visualização do gráfico predito pela função ajustada pode ser realizada por meio de gráficos de superfícies de resposta. Esta representação gráfica é uma superfície ndimensional no espaço $n+1$ dimensional. De forma usual, uma representação bidimensional de um gráfico tridimensional pode ser obtida. Então, se há três ou mais variáveis, a visualização do gráfico é possível apenas se uma ou mais variáveis são fixadas em um determinado valor. ${ }^{3,16,52}$

Para modelos quadráticos, o ponto crítico pode ser caracterizado como máximo, mínimo ou sela (Figura 2). É possível calcular as coordenadas do ponto crítico através das primeiras derivadas da função matemática que descreve a superfície de resposta em relação a cada variável e igualando-as a zero. A função quadrática obtida para otimização de duas variáveis descrita abaixo é usada como exemplo:
As superfícies de resposta geradas por modelo lineares podem ser usadas para indicar a direção na qual o planejamento original deve ser deslocado para que as condições ótimas sejam atingidas. Contudo, se a região experimental não pode ser deslocada devido às razões físicas ou 
instrumentais, o pesquisador deve encontrar, por inspeção visual, as melhores condições dentro do espaço experimental estudado. Muitas vezes, a superfície quadrática obtida também pode indicar um ótimo localizado fora da região estudada indicando a direção no qual o planejamento deve ser deslocado, se possível.

\section{Programas estatísticos usados em planejamento experimental}

Diversos programas computacionais ou pacotes estatísticos que disponibilizam ferramentas para uso em Quimiometria (incluindo a geração e análise de planejamentos experimentais) estão disponíveis no mercado. Os programas podem ser encontrados para as plataformas Windows, Mac OS, Linux, entre outros sistemas operacionais ${ }^{66}$. Segundo Ramos e Álvarez-Coque ${ }^{67}$, pode-se dividi-los nos seguintes grupos:

(1) Os pacotes estatísticos de aplicação geral em diversos ramos da Ciência, como Statistica, SAS (Statistical Analysis System), Statigraphics, Minitab, Design Expert, Modde, Quantum XL, entre outros. São ambientes de trabalho com opções que englobam um grande número de ferramentas estatísticas programadas para fins diversos, e podem perfeitamente ser usados para tratar dados quimiométricos.

(2) Os pacotes de Quimiometria especialmente desenvolvidos para os tratamentos de dados químicos como, por exemplo, The Unscrambler. Em geral, esses programas não contemplam todas as ferramentas estatísticas e centram sua atenção nas ferramentas mais utilizadas perdendo seu enfoque geral em nome da simplicidade, rapidez e comodidade.

(3) Os ambientes assistidos para o desenvolvimento de ferramentas estatísticas como MATLAB, Scilab e Octave. Neste caso, se disponibilizam opções de ferramentas básicas (como vetores e matrizes) para que $o$ usuário crie seu próprio programa. É necessário certo grau de treinamento para usá-los. No entanto, dominar um ambiente desse tipo possibilita desenvolver novas ferramentas quimiométricas e modificar as existentes.

(4) As planilhas de cálculo como Excel, Calc, Quattro Pro, Origin, entre outros. Os três primeiros são encontrados em pacotes básicos como no Microsoft Office, no LibreOffice e no Corel Office respectivamente. Eles possuem uma extensa relação de ferramentas estatísticas e podem servir de plataforma para entrada de dados e equações, realização de cálculos necessários para aplicação dos planejamentos experimentais, além de proporcionarem excelente visualização gráfica dos resultados.

Esses programas podem apresentar uma variação de preços a depender da versão adquirida que pode ser da mais básica a mais completa. Em uma busca realizada para alguns programas, em sites de venda especializados, revelou que seus preços podem variar de U\$ 100,00 a U\$ $4.000,00$. Devido aos custos relativamente altos envolvidos na aquisição de pacotes computacionais estatísticos há, atualmente, o aumento de demanda por softwares livres. Esses programas são abertos ao uso público e podem ser usados gratuitamente no desenvolvimento de um trabalho científico que envolva planejamento experimental e análise de dados.

Entre os programas gratuitos pode-se citar o pacote de algoritmos disponíveis nos websites Chemkeys ${ }^{68}$ e do Laboratório de Quimiometria Teórica e Aplicada do Instituto de Química (UNICAMP, Brasil), o Chemoface ${ }^{69}$ (UFLA, Brasil), o Action ${ }^{70}$ (Statcamp, Brasil), entre outros. Além da gratuidade, somam-se às vantagens do uso de softwares livres: a geração de gráficos com alta qualidade e definição, a realização de cálculos e simulações confiáveis podem ser feitos em poucos segundos se equiparando aos programas comerciais, a agregação de melhorias pela contribuição espontânea de desenvolvedores e usuários, geralmente podem rodar em ambientes de planilhas 
desenvolvidas por outras empresas ou instituições como o Excel, o Calc, etc, e contribuem na difusão dos conhecimentos na área de Quimiometria atuando como uma ferramenta auxiliar de ensino-aprendizagem nas aulas de disciplinas que envolvem planejamento experimental $^{66}$.

\section{Exemplos da aplicação da MSR na otimização de métodos analíticos}

Serão comentados três exemplos para ilustrar o uso da metodologia de superfícies de respostas na otimização de métodos analíticos.

\section{Exemplo 1}

Santelli et al. ${ }^{63}$ utilizaram metodologia de superfícies de resposta para otimizar a programação de temperatura em espectrometria de absorção atômica com forno de grafite (GFAAS) para determinação de vanádio em amostras de combustíveis após sua emulsificação com o surfactante Triton X-100.

As variáveis (temperatura e tempo de pirólise e temperatura de atomização) foram otimizadas usando-se uma matriz de Doehlert para três variáveis geradas pela projeção plana apresentada na Figura 1c. O domínio experimental é apresentado na Tabela 3. Para permitir a estimativa do erro experimental, as condições experimentais correspondentes ao ponto central foram realizadas em triplicata. A estes dados experimentais, foram ajustados o modelo linear e o modelo quadrático.

A Análise de variância (ANOVA) indicou que o modelo linear não é adequado para a descrição da região experimental estudada, pois o modelo apresenta falta de ajuste $(p=$ $0,0127<0,05)$. No entanto, há uma boa concordância entre os valores de absorbância predita pelo modelo e os valores encontrados experimentalmente quando se ajusta um modelo quadrático aos dados obtidos $(\mathrm{p}=$ $0.9999>0.05)$.

Outra forma de avaliar a qualidade dos modelos ajustados é observando-se os gráficos de resíduos. A Figura 3a mostra o comportamento dos resíduos deixados pelo ajuste do modelo linear. Pode-se observar que, para a maioria dos pontos experimentais, os resíduos deixados são muito altos e apresentam um comportamento que indica a necessidade de termos quadráticos na equação. Na Figura $3 b$ tem-se o gráfico de resíduos para o modelo quadrático. Observa-se que os resíduos deixados pelo modelo quadrático são menores e se distribuem aleatoriamente sugerindo que os mesmos seguem uma distribuição normal. Os gráficos de valores observados versus valores preditos (Figuras $4 a$ e $4 b)$ também reforçam que o modelo quadrático é superior ao linear na descrição do comportamento dos dados. Portanto, este modelo pode ser usado para descrever o comportamento da resposta de acordo com a variação dos níveis das variáveis estudadas e ele foi escolhido para o cálculo das coordenadas do ponto ótimo.

(a) 

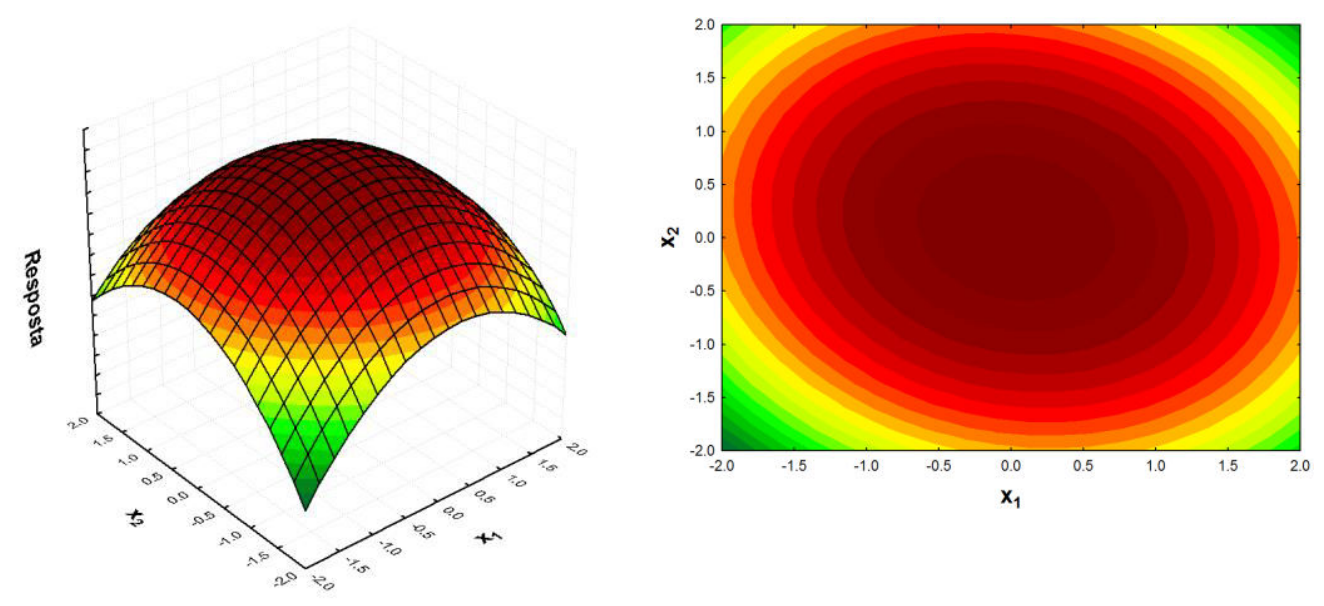

(b)
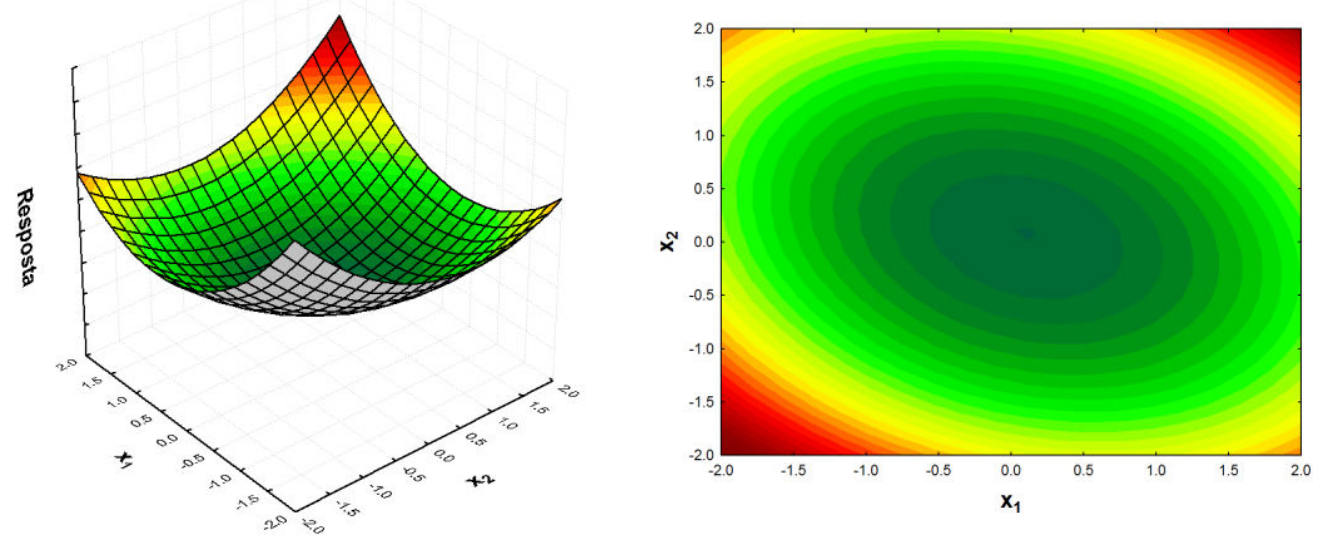

(c)
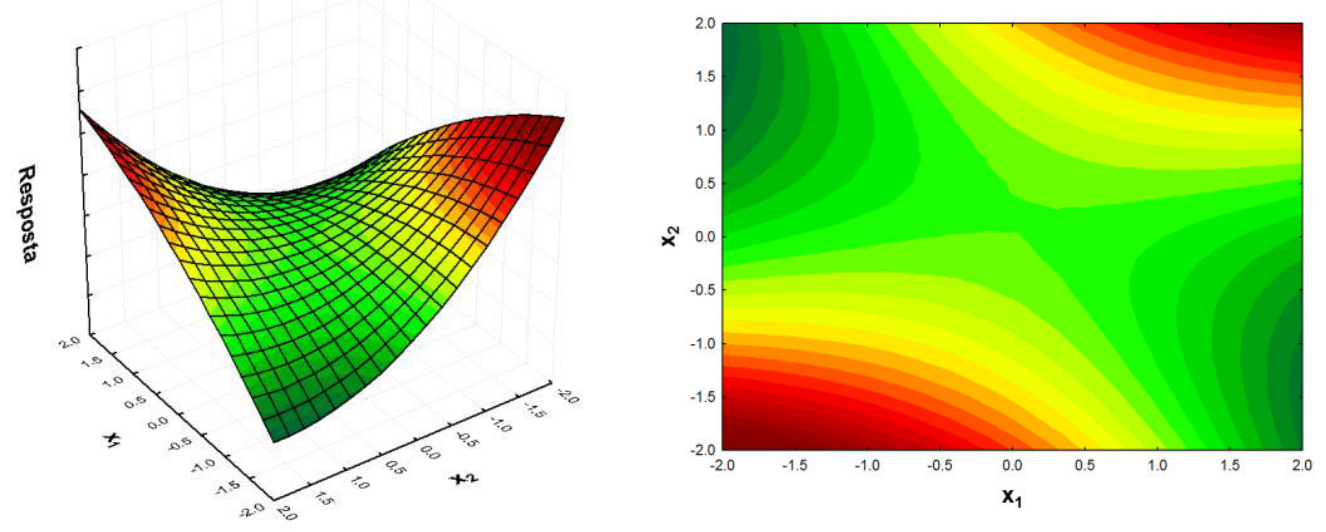

Figura 2. Perfis de superfícies de respostas e curvas de níveis mais comuns: (a) superfície com ponto de máximo; (b) superfície com ponto de mínimo e (c) superfície com ponto de sela 

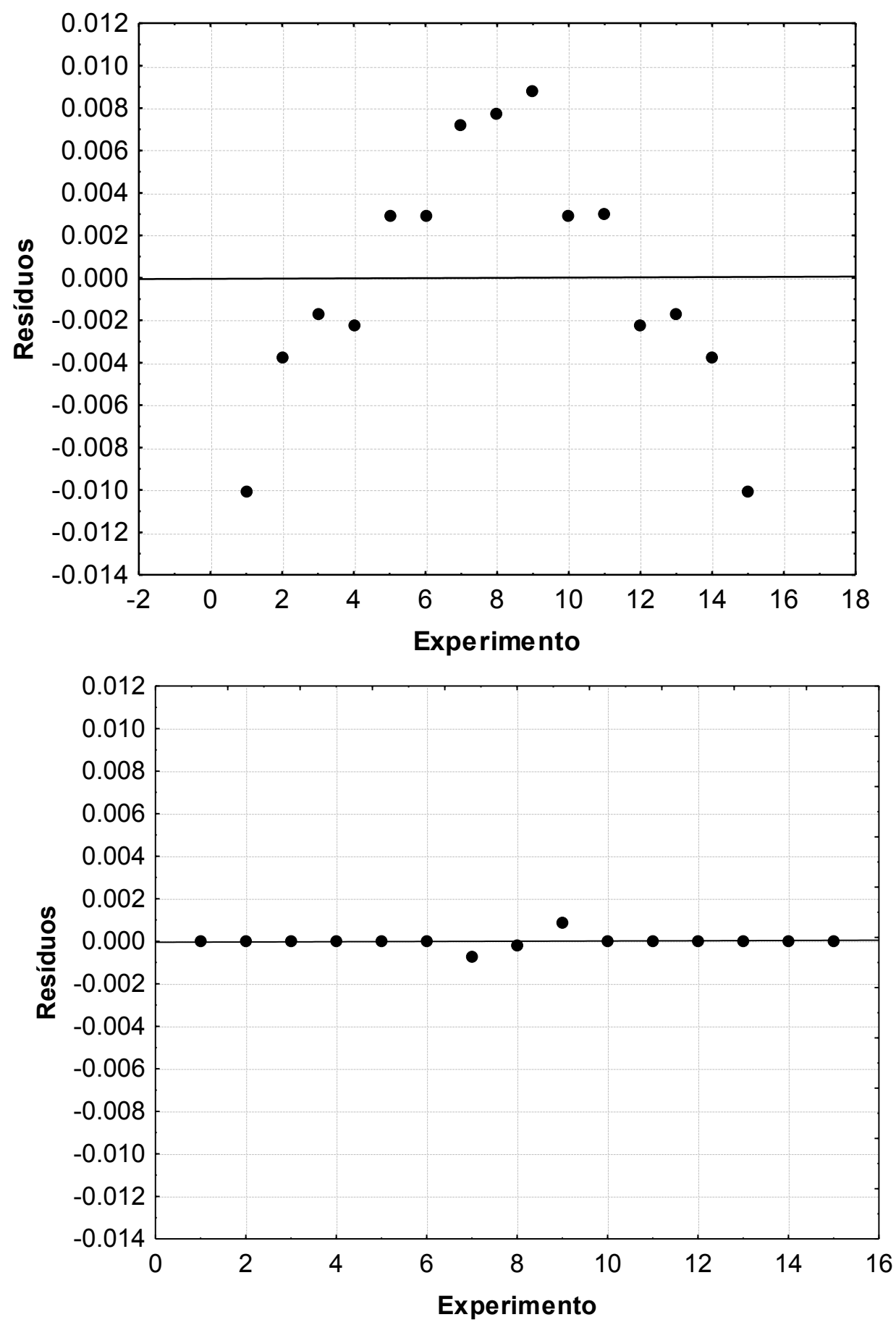

Figura 3. Gráfico de resíduos (a) para o modelo linear; (b) para o modelo quadrático 
Novaes, C. G. et al.
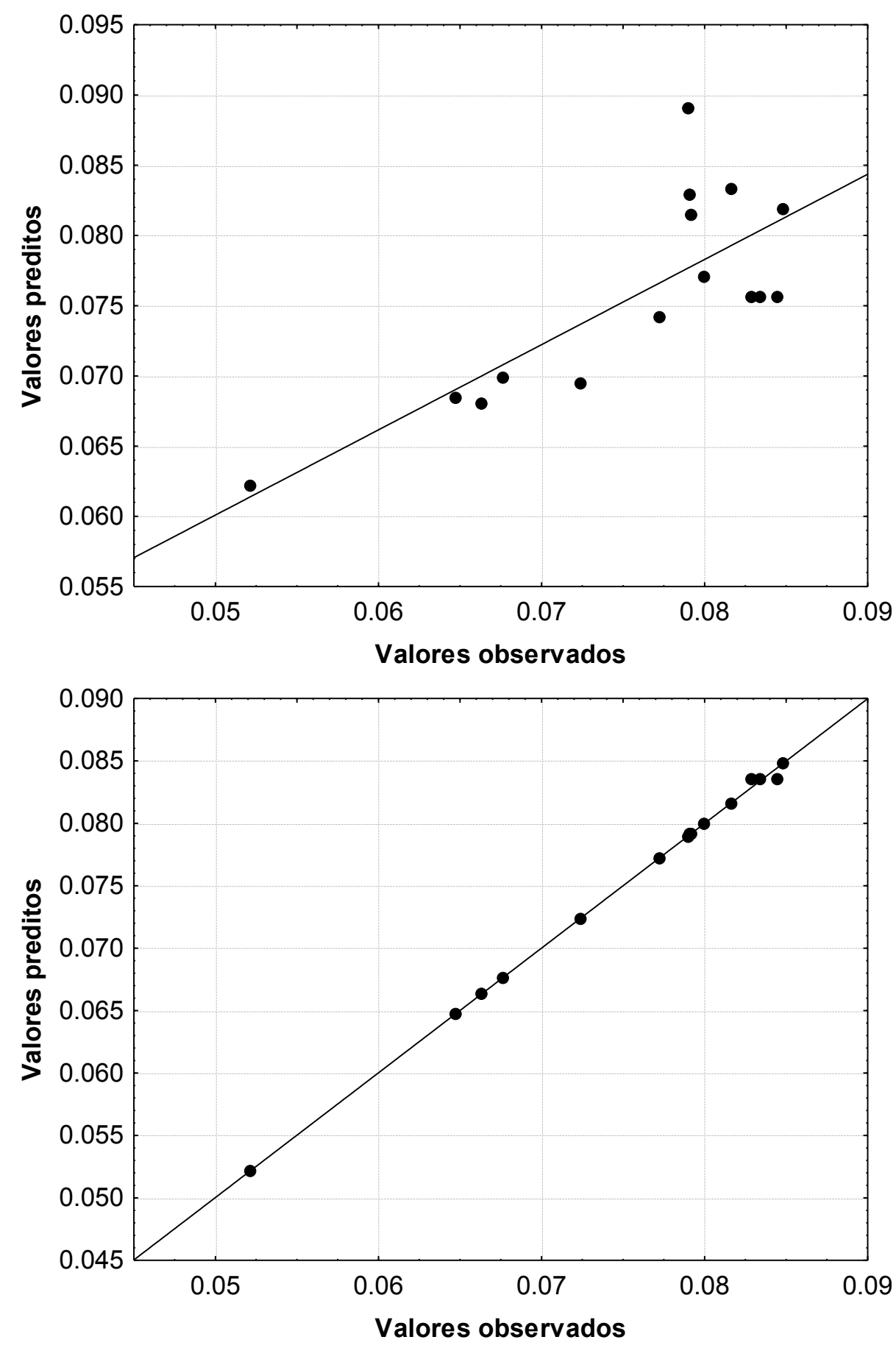

Figura 4. Gráfico de valores preditos X valores observados: (a) para o modelo linear e (d) para o modelo quadrático 
Tabela 3. Planejamento experimental Doehlert para otimização do programa de temperatura em GFAAS para determinação de vanádio em emulsões de combustíveis

\begin{tabular}{|c|c|c|c|c|}
\hline Exp. & $\mathbf{x}$ & $\mathbf{y}$ & $\mathbf{z}$ & $\mathbf{R}$ \\
\hline $\mathbf{1}$ & $2800(1)$ & $1200(0)$ & $30(0)$ & 0,0790 \\
\hline $\mathbf{2}$ & $2700(0,5)$ & $1100(-0,5)$ & $20(-0,707)$ & 0,0791 \\
\hline $\mathbf{3}$ & $2700(0,5)$ & $1100(-0,5)$ & $40(0,707)$ & 0,0816 \\
\hline $\mathbf{4}$ & $2700(0,5)$ & $1300(0,5)$ & $20(-0,707)$ & 0,0792 \\
\hline $\mathbf{5}$ & $2700(0,5)$ & $1300(0,5)$ & $40(0,707)$ & 0,0848 \\
\hline $\mathbf{6}$ & $2600(0)$ & $1000(-1)$ & $30(0)$ & 0,0800 \\
\hline $\mathbf{7}$ & $2600(0)$ & $1200(0)$ & $30(0)$ & $0,0829 / 0,0834 / 0,0845$ \\
\hline $\mathbf{8}$ & $2600(0)$ & $1400(1)$ & $30(0)$ & 0,0772 \\
\hline $\mathbf{9}$ & $2500(-0,5)$ & $1100(-0,5)$ & $20(-0,707)$ & 0,0724 \\
\hline $\mathbf{1 0}$ & $2500(-0,5)$ & $1100(-0,5)$ & $40(0,707)$ & 0,0676 \\
\hline $\mathbf{1 1}$ & $2500(-0,5)$ & $1300(0,5)$ & $20(-0,707)$ & 0,0663 \\
\hline $\mathbf{1 2}$ & $2500(-0,5)$ & $1300(0,5)$ & $40(0,707)$ & 0,0647 \\
\hline $\mathbf{1 3}$ & $2400(-1)$ & $1200(0)$ & $30(0)$ & 0,0521 \\
\hline
\end{tabular}

OBS: os experimentos foram realizados em ordem aleatória e os valores codificados são apresentados entre parênteses; R: resposta (absorbância); $x$ : temperatura de atomização, y: temperatura de pirólise e z: tempo de pirólise.

A equação (14) relaciona as três variáveis estudadas com a resposta analítica $(R)$, onde $R$ é a absorbância, $x$, y e z são a temperaturas de atomização, a temperatura de pirólise e o tempo de pirólise, respectivamente:

$$
\begin{aligned}
& R=0,0836( \pm 0,0005)+0,0134 x( \pm 0,0004)-0,0181 x^{2}(0,0008)-0,0014 y( \pm 0,0004)- \\
& 0,0050 y^{2}( \pm 0,0008)+0,0003 z( \pm 0,0005)-0,0067 z^{2}( \pm 0,0009)+0,0062 x y( \pm 0,0011)+ \\
& 0,0051 x z( \pm 0,0008)+0,0022 y z( \pm 0,0008)
\end{aligned}
$$

Na equação 14 , os termos em negrito são significativos em um nível de confiança de $95 \%$ e os outros termos podem ser eliminados da equação para facilitar o cálculo do ponto crítico. A superfície encontrada é definida como uma superfície de máximo. É possível calcular as coordenadas do ponto crítico por meio das primeiras derivadas de cada variável em relação à resposta dessa função matemática e igualando-as a zero.

$$
\begin{aligned}
& \frac{\partial R}{\partial x}=0,0134-0,0362 x+0,062 y+0,0051 z=0 \\
& \frac{\partial R}{\partial y}=-0,0014+0,0062 x-0,0100 y+0,0022 z=0
\end{aligned}
$$




$$
\frac{\partial R}{\partial z}=0,0003+0,0051 x+0,0022 y-0,0134 z=0
$$

Para calcular a coordenada do ponto crítico é necessário resolver este sistema de primeiro grau, formado pelas equações 15 , 16 e 17. A resolução do sistema de equações é feita calculando-se a primeira derivada das equações que descrevem as superfícies de resposta em relação a cada componente permite calcular esses valores ótimos. Nesse caso, para encontrar os valores exatamente iguais aos calculados pelo programa estatístico, não eliminou-se os termos não significativos.

Como é possível utilizar apenas três eixos para representar graficamente as superfícies e um dos eixos deve ser reservado para a resposta, para três variáveis ter-se-ão três representações gráficas (derivada pela combinação das variáveis de duas em duas) para esta superfície de resposta.

A superfície apresenta ponto de máximo como ponto crítico. As coordenadas destes pontos são as condições experimentais que geram a maior resposta para o sistema estudado. Desta forma, os valores ótimos encontrados para a realização das extrações foram: 0,$43 ; 0,17$ e 0,22 em valores codificados, os quais correspondem aos valores reais de $2690 \circ \mathrm{C}, 1200 \circ \mathrm{C}$ e 35 segundos para a temperatura de atomização, temperatura de pirólise e tempo de pirólise, respectivamente.
As condições experimentais encontradas como ótimas foram aplicadas na determinação de vanádio em amostras de derivados de petróleo permitindo a quantificação deste metal com boas características analíticas. Os valores experimentais encontrados pela aplicação das condições ótimas foram concordantes com aqueles previsto pelo planejamento experimental.

\section{Exemplo 2}

Santos et al. ${ }^{13}$ utilizaram um planejamento Box-Behnken, mostrado na Figura 1c, para otimizar três variáveis (tempo de sonicação, concentração da solução ácida extratora e tamanho da partícula) envolvidas em um procedimento de extração assistida por ultrassom na análise de amostras de sementes de feijão. As respostas obtidas foram as percentagens de extração de nove elementos ( $\mathrm{Ba}, \mathrm{Ca}, \mathrm{Cu}, \mathrm{Fe}, \mathrm{K}, \mathrm{Mg}, \mathrm{Mn}, \mathrm{Sr}$ e $\mathrm{Zn}$ ) tomadas em comparação com $\mathrm{O}$ procedimento da digestão assistida por micro-ondas. Para permitir a otimização simultânea da extração dos metais estudados, todas as respostas obtidas foram combinadas na resposta global (RG), calculada, para cada experimento, através da seguinte expressão:

$$
R G=\left[R\left(x_{1}\right) / M R\left(x_{1}\right)+R\left(x_{2}\right) / M R\left(x_{2}\right)+\ldots+R\left(x_{n}\right) / M R\left(x_{n}\right)\right]
$$

Onde $R\left(x_{n}\right)$ é a resposta para um elemento em um experimento particular e $M R\left(x_{n}\right)$ é a maior resposta no conjunto para aquele elemento $\mathrm{n}$.

A matriz experimental, juntamente com a resposta global, são apresentadas na Tabela 4. Os experimentos foram realizados em duplicata (e o ponto central em triplicata) para permitir a avaliação do erro experimental. 
Tabela 4. Matriz experimental de Box-Behnken e resultados (resposta global) para otimização da extração assistida por ultrassom

\begin{tabular}{|c|c|c|c|c|}
\hline Exp* & $\begin{array}{c}\text { TS } \\
(\mathbf{m i n})\end{array}$ & $\begin{array}{c}\boldsymbol{C} \\
\left(\mathbf{m o l ~ L}^{-1}\right)\end{array}$ & $\begin{array}{c}\boldsymbol{T P} \\
(<\mu \mathrm{m})\end{array}$ & Resposta Global \\
\hline 1 & $5(-1)$ & $1,5(0)$ & $100(-1)$ & $6,89 / 6,55$ \\
\hline 2 & $15(0)$ & $2,5(1)$ & $500(1)$ & $7,62 / 7,63$ \\
\hline 3 & $15(0)$ & $0,5(-1)$ & $500(1)$ & $7,99 / 7,27$ \\
\hline 4 & $15(0)$ & $1,5(0)$ & $300(0)$ & $7,80 / 8,39$ \\
\hline 5 & $25(1)$ & $0,5(-1)$ & $300(0)$ & $8,14 / 8,19$ \\
\hline 7 & $5(-1)$ & $1,5(0)$ & $500(1)$ & $7,27 / 8,20$ \\
\hline 10 & $15(0)$ & $0,5(-1)$ & $100(-1)$ & $5,43 / 5,13$ \\
\hline 11 & $15(0)$ & $2,5(1)$ & $100(-1)$ & $7,50 / 6,81$ \\
\hline 12 & $25(1)$ & $2,5(1)$ & $300(0)$ & $7,33 / 8,46$ \\
\hline 13 & $15(0)$ & $1,5(0)$ & $300(0)$ & $8,11 / 8,13 / 8,42$ \\
\hline 14 & $5(-1)$ & $0,5(-1)$ & $300(0)$ & $5,23 / 6,93$ \\
\hline 16 & $5(-1)$ & $2,5(1)$ & $300(0)$ & $8,39 / 8,26$ \\
\hline 22 & $25(1)$ & $1,5(0)$ & $500(1)$ & $8,82 / 8,54$ \\
\hline 23 & $25(1)$ & $1,5(0)$ & $100(-1)$ & $6,23 / 6,31$ \\
\hline
\end{tabular}

*Os experimentos foram realizados em ordem aleatória. TS, tempo de sonicação; C, concentração e TP, tamanho da partícula.

Foram ajustados modelos (linear e estatisticamente significativo, pois o valor quadrático) à resposta global e ANOVA calculado para $F(14,05)$ foi maior que o valor (Tabela 5) foi usada para acessar a tabelado $\left(F_{9,19}=2,42\right)$ e não apresentou falta adequação dos modelos ajustados. O modelo de ajuste pois o valor calculado de $F(0,68)$ foi quadrático mostrou-se superior, sendo menor que o tabelado $\left(F_{3,16}=3,24\right)$.

Tabela 5. Análise de variância para (a) modelo linear e (b) modelo quadrático ajustados à resposta global para um nível de confiança de $95 \%$

\begin{tabular}{|c|c|c|c|c|c|}
\hline \multicolumn{7}{|c|}{ (a) Modelo linear } \\
\hline Fonte de variação & SQ & g.l. & MQ & $\mathrm{F}_{\text {calc. }}$ & $\mathrm{F}_{\text {tab. }}$ \\
\hline Regressão & 14,60164 & 3 & 4,86721 & 8,182 & 2,99 \\
\hline Resíduo & 14,87154 & 25 & 0,59486 & & \\
\hline Falta de ajuste & 11,45044 & 9 & 1,272271 & 5,950 & 2,54 \\
\hline Erro puro & 3,42110 & 16 & 0,213819 & & \\
\hline Total & 29,47318 & 28 & & & \\
\hline \multicolumn{7}{|c|}{ (b) Modelo quadrático } \\
\hline Fonte de variação & SQ & g.l. & MQ & $\mathrm{F}_{\text {calc. }}$ & $\mathrm{F}_{\text {tab. }}$ \\
\hline Regressão & 25,637 & 9 & 2,8486 & 14,05 & 2,42 \\
\hline Resíduo & 3,856 & 19 & 0,2028 & & \\
\hline Falta de ajuste & 0,4333 & 3 & 0,1442 & 0,6756 & 3,24 \\
\hline Erro puro & 3,4204 & 16 & 0,2137 & & \\
\hline Total & 29,491 & 28 & & & \\
\hline
\end{tabular}

$\mathrm{SQ}$, soma quadrática; g.I., grau de liberdade; $\mathrm{MQ}$, média quadrática 
A superfície ajustada foi caracterizada como superfície de sela e, portanto, as coordenadas do ponto crítico (um ponto de inflexão) não fornecem nem a maior nem a menor resposta. Desta forma, as condições de maior extração serão encontradas por inspeção visual das superfícies (ou curvas de níveis). As seguintes condições foram tomadas como ótimas: 15 minutos de exposição à energia de ultrassom, tamanho de partícula menor que $300 \mu \mathrm{m}$ e utilização de solução extratora com concentração de $1,5 \mathrm{~mol} \mathrm{~L}^{-1}$. Quando as extrações são feitas nestas condições, encontram-se percentagens de extração entre 92 e 106\%. O elemento $\mathrm{Fe}$ não apresentou extrações quantitativas e foi posteriormente excluído do estudo.

\section{Exemplo 3}

Barros et $\mathrm{al}^{64}$ usaram planejamento Doehlert e função de desejabilidade na otimização de um método com base na extração assistida por ultrassom para determinação de cobre, manganês, níquel e zinco por espectrometria de absorção atômica com chama (FAAS) em amostras de ração para nutrição de frangos. Os resultados são apresentados na Tabela 6. As variáveis otimizadas foram: massa da amostra, tempo de sonicação e concentração final da mistura extratora forma pelos ácidos nítrico, clorídrico e acético. As funções de desejabilidade individuais utilizadas tiveram como objetivo maximizar as respostas (Percentagem de extração de cada metal). 0 modelo quadrático ajustado à desejabilidade global (Equação 19) mostrou-se superior ao linear e, portanto, ele foi escolhido para indicar as condições ótimas para a extração simultânea dos metais. Nessa equação, os valores em negrito são significativos em um nível de confiança de 95\%. Superfícies de respostas foram modeladas usando-se a desejabilidade global como resposta. A Figura 5 mostra os perfis de desejabilidades individuais e globais nas condições estabelecidas para esta análise. A desejabilidade global máxima atingida foi igual a 0,8176. Este valor pode variar de 0 a 1. Quanto mais próximo da unidade for este valor, melhor para a otimização simultânea. Esse valor indica que os ótimos individuais correspondentes a cada resposta estão próximos entre si e que pode haver uma condição experimental que os satisfaçam simultaneamente apesar de se perder um pouco na capacidade de extração para cada metal. Este é o preço a ser pago na otimização simultânea ainda que ele seja pequeno. Desta forma, as melhores condições para extração dos metais são 3,2 $\mathrm{mol} \mathrm{L}^{-1}$ (concentração do ácido); 0,24 $\mathrm{g}$ (massa da amostra) e $18 \mathrm{~min}$ (tempo de sonicação). Usando-se estes parâmetros, boas recuperações podem ser obtidas usando-se o método desenvolvido.

$$
\begin{gathered}
\mathrm{DG}=0,756( \pm 0,05)-\mathbf{0 , 0 0 3}( \pm \mathbf{0 , 0 3})(\mathrm{AC})-0,148( \pm 0,06)(\mathrm{AC})^{2}-0,261( \pm 0,03)(\mathrm{SM})- \\
0,518( \pm 0,06)(\mathrm{SM})^{2}+0,142( \pm 0,03)(\mathrm{ST})-0,481( \pm 0,07)(\mathrm{ST})^{2}-\mathbf{0 , 1 3 2}( \pm \mathbf{0 , 0 7}) \\
(\mathrm{AC})(\mathrm{SM})-\mathbf{0 , 0 4 1}( \pm \mathbf{0 , 0 5})(\mathrm{AC})(\mathrm{ST})-\mathbf{0 , 0 1 6}( \pm \mathbf{0 , 0 5})(\mathrm{SM})(\mathrm{ST})
\end{gathered}
$$


CA
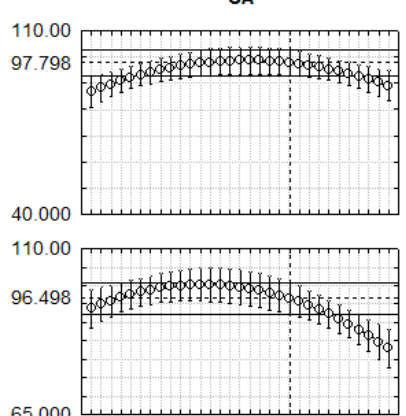

65.000
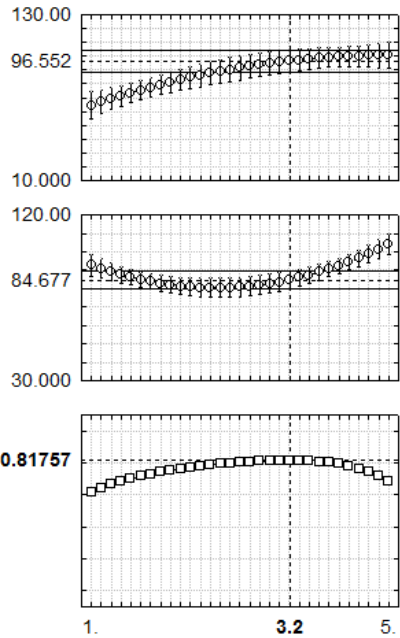

MA
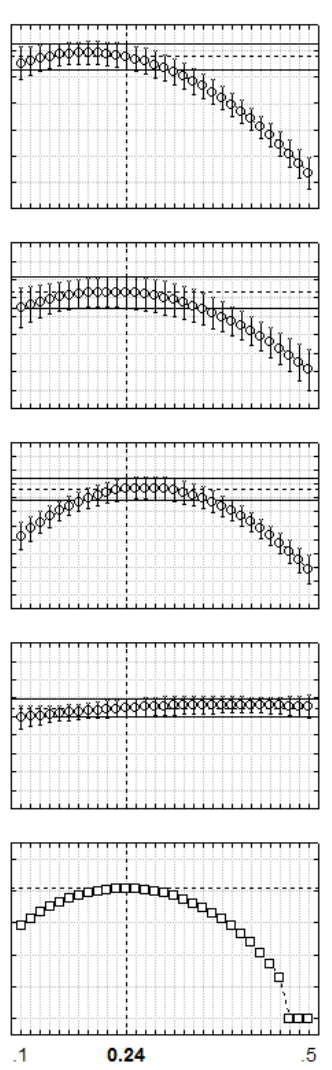
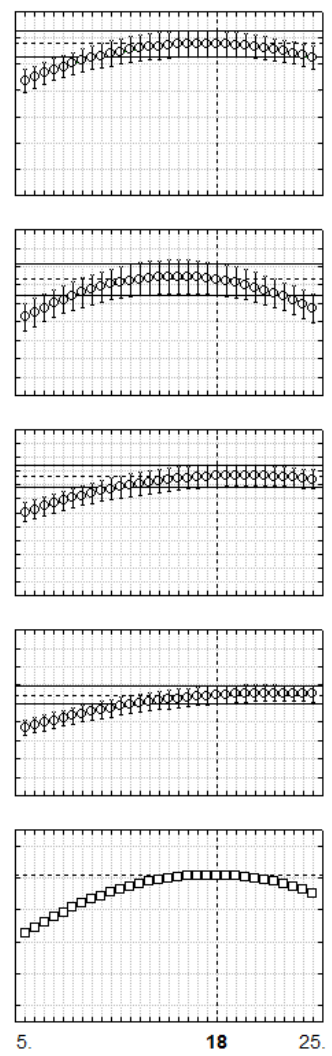

Desirability
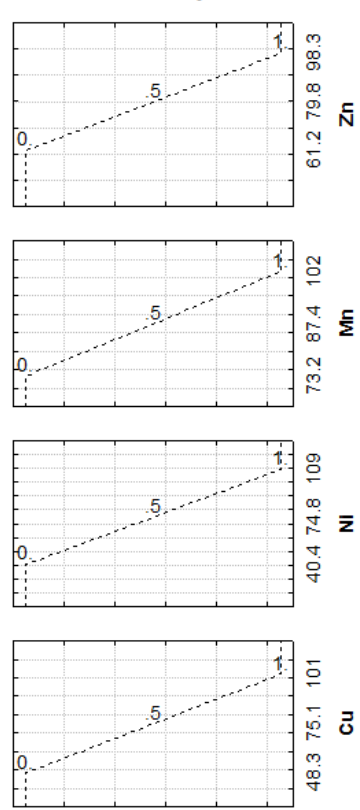

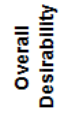

Figura 5. Perfis para os valores preditos das desejabilidades individuais e global na otimização dos dados gerados usando-se planejamento Doehlert na otimização do método para extração de metais usando-se energia de ultrassom 


\section{$\mathrm{R} / \mathrm{q}$}

Tabela 6. Planejamento Doehlert e respostas (percentage de recuperação de cobre, manganês, níquel e zinco) para otimização das variáveis de método: concentração do ácido (CA), massa da amostra (MA) e tempo de sonicação (TS)

\begin{tabular}{|c|c|c|c|c|c|c|c|}
\hline \multicolumn{3}{|c|}{ Variáveis } & \multicolumn{4}{|c|}{ Extração (\%) } & \multirow{2}{*}{ Desejabilidade Globa } \\
\hline CA & MA & TS & $\mathrm{Cu}$ & $M n$ & $\mathbf{N i}$ & $\mathrm{Zn}$ & \\
\hline $3(0)$ & $0,5(1)$ & $15(0)$ & $71,08 / 64,44$ & $79,60 / 76,98$ & $44,33 / 53,05$ & $61,95 / 62,75$ & $0,0991 / 0,1299$ \\
\hline $2(-0,5)$ & $0,4(0,5)$ & $25(0,707)$ & $66,56 / 62,74$ & $90,63 / 80,87$ & 69,83 / 61,09 & $75,38 / 78,03$ & $0,4290 / 0,3145$ \\
\hline $4(0,5)$ & $0,4(0,5)$ & $5(-0,707)$ & 70,49 / 63,70 & 73,28 / 75,39 & $44,21 / 43,70$ & 68,10 / 61,79 & $0,0035 / 0,0612$ \\
\hline $3(0)$ & $0,3(0)$ & $15(0)$ & $82,10 / 72,69$ & 98,19 / 98,76 & 94,47 / 88,65 & 98,37 / 92,22 & 0,8124 / 0,6993 \\
\hline $5(1)$ & $0,3(0)$ & $15(0)$ & $101,9 / 100,79$ & 82,12 / 83,63 & $95,63 / 109,18$ & 82,89 / 94,90 & 0,6179 / 0,7546 \\
\hline $2(-0,5)$ & $0,2(-0,5)$ & $5(-0,707)$ & 75,76 / 68,66 & 91,32 / 86,89 & 65,65 / 66,50 & 79,90 / 84,50 & 0,4949 / 0,4561 \\
\hline $2(-0,5)$ & $0,2(-0,5)$ & $25(0,707)$ & 79,55 / 86,79 & 93,88 / 89,41 & 83,15 / 62,13 & $94,91 / 93,79$ & 0,6989 / 0,5799 \\
\hline
\end{tabular}

NOTA: Os valores codificados estão entre parênteses 


\section{Conclusões}

A aplicação de Metodologia de Superfícies de Resposta (MSR) na otimização de variáveis de processo para o desenvolvimento de métodos analíticos está, nos dias atuais, largamente difundida e consolidada principalmente devido às suas vantagens em relação à clássica otimização univariada, com a geração de informações importantes utilizando um pequeno número de experimentos e a possibilidade de avaliação dos efeitos de interação entre as variáveis e a resposta.

\section{Agradecimentos}

Os autores deste artigo agradecem ao Conselho Nacional de Desenvolvimento Científico e Tecnológico (CNPq), Fundação de Amparo à Pesquisa do estado da Bahia (FAPESB) e a Coordenação de Aperfeiçoamento de Pessoal de Nível Superior (CAPES) pelo suporte financeiro.

\section{Referências Bibliográficas}

${ }^{1}$ Araújo, P. W.; Brereton, R. G. Experimental design II. Optimization. TrAC, Trends in Analytical Chemistry 1996, 15, 63. [CrossRef]

${ }^{2}$ Bezerra, M. A.; Santelli, R. E.; Oliveira, E. P.; Villar, L. S.; Escaleira, L. A. Response surface methodology (RSM) as a tool for optimization in analytical chemistry. Talanta 2008, 76, 965. [CrossRef] [PubMed]

${ }^{3}$ Lundstedt, T.; Seifert, E.; Abramo, L.; Thelin, B.; Nystrom, A.; Pettersen, J.; Bergman, R. Experimental design and optimization. Chemometrics and Intelligent Laboratory Systems 1998, 42, 3. [CrossRef]

${ }^{4}$ Ferreira, S. L. C.; Bruns, R. E.; Ferreira, H. S.; Matos, G. D.; David, J. M.; Brandão, G. C.; Silva, E. G. P.; Portugal, L. A.; Reis, P. S.;
Souza, A. S.; Santos, W. N. L. Box-Behnken design: An alternative for the optimization of analytical methods. Analytica Chimica Acta 2007, 597, 179. [CrossRef] [PubMed]

${ }^{5}$ Brown, S. D.; Tauler, R.; Walczak, B.; Comprehensive Chemometrics: Chemical and Biochemical Data Analysis, vol. 1, $1^{\text {st }}$ ed., Elsevier: Amsterdam, 2009

${ }^{6}$ Bruns, R. E.; Scarminio, I. S.; Neto, B. B.; Statistical Design - Chemometrics, $1^{\text {st }}$ ed., Elsevier Science: Amsterdam, 2006.

${ }^{7}$ Hanrahan, G.; Lu, K. Application of Factorial and Response Surface Methodology in Modern Experimental Design and Optimization. Critical Reviews in Analytical Chemistry 2006, 36, 141. [CrossRef]

${ }^{8}$ Orlandini, S.; Gotti, R.; Furlanetto, S. Multivariate optimization of capillary electrophoresis methods: A critical review. Journal of Pharmaceutical and Biomedical Analysis 2014, 87, 290. [CrossRef] [Pubmed]

${ }^{9}$ Sivertsen, E.; Bjerke, F.; Almøy, T.; Segtnan, V.; Naes, T. Multivariate optimization by visual inspection. Chemometrics and Intelligent Laboratory Systems 2007, 85, 110. [CrossRef]

${ }^{10}$ Costa, N. R.; Lourenço, J.; Pereira, Z. L. Desirability function approach: A review and performance evaluation in adverse conditions. Chemometrics and Intelligent Laboratory Systems 2011, 107, 234. [CrossRef]

${ }^{11}$ Calado, V.; Montgomery, D.; Planejamento de experimentos usando o Statistica, 1st Ed., E-papers: Rio de Janeiro, 2003. [CrossRef]

${ }^{12}$ Candioti, L. V.; Zan, M. M.; Camara, M. S.; Goicoechea, H. C. Experimental design and multiple response optimization. Using the desirability function in analytical methods development. Talanta 2014, 124, 123. [CrossRef] [PubMed]

${ }^{13}$ Santos, W. P. C.; Castro, J. T.; Bezerra, M. A.; Fernandes, A. P.; Ferreira, S. L. C.; Korn, M. G. A. Application of multivariate optimization in the development of an ultrasound-assisted extraction procedure for 
multielemental determination in bean seeds samples using ICP OES. Microchememical Journal 2009, 91, 153. [CrossRef]

${ }^{14}$ Bezerra, M. A.; Bruns, R. E.; Ferreira, S. L. C. Statistical design-principal component analysis optimization of a multiple response procedure using cloud point extraction and simultaneous determination of metals by ICP OES. Analytica Chimica Acta 2006, 580, 251. [CrossRef] [PubMed]

${ }^{15}$ Otto, M.; Chemometrics, 2nd ed., Willey VCH: New York, 2007.

${ }^{16}$ Ferreira, S. L. C.; Santos, W. N. L.; Quintella, C. M.; Neto, B. B.; Bosque-Sendra, J. A. Doehlert matrix: a chemometric tool for analytical chemistry-review. Talanta 2004, 63, 1061. [CrossRef] [Pubmed]

17 Massart, D. L.; Vandeginste, B. G. M.; Buydens, J. M. C.; Jong, S.; Lewis, P. J.; Smeyers-Verberke, J.; Handbook of Chemometrics and Qualimetrics, 1st ed., Elsevier: Amsterdam, 1997.

18 Tarley, C. R. T.; Silveira, G.; Santos, W. N. L.; Matos, G. D.; Silva, E. G. P.; Bezerra, M. A.; Miró, M.; Ferreira, S. L. C. Chemometric tools in electroanalytical chemistry: Methods for optimization based on factorial design and response surface methodology. Microchemical Journal 2009, 92, 58. [CrossRef]

${ }^{19}$ Hibbert, D. B. Experimental design in chromatography: a tutorial review. Journal of Chromatography B 2012, 910, 2. [CrossRef] [PubMed]

${ }^{20}$ Cespon-Romero, R. M.; Yebra-Biurrun, M. C. Determination of trace metals in urine with an on-line ultrasound-assisted digestion system combined with a flow-injection preconcentration manifold coupled to flame atomic absorption spectrometry. Analytical Chimica Acta 2008, 609, 184. [CrossRef] [PubMed]

${ }^{21}$ Silva, L. O.; Leão, D. J.; Debora, C. S.; Matos, G. D.; Andrade, J. B.; Ferreira, S. L. C. Determination of copper in airborne particulate matter using slurry sampling and chemical vapor generation atomic absorption spectrometry. Talanta 2014, 127, 140. [CrossRef] [PubMed]

${ }^{22}$ Ebrahimzadeh, H.; Behbahani, M.; Yamini, Y.; Adlnasab, L.; Asgharinezhad, A. A. Optimization of $\mathrm{Cu}(\mathrm{II})$-ion imprinted nanoparticles for trace monitoring of copper in water and fish samples using a BoxBehnken design. Reactive and Functional Polymer 2013, 73, 23. [CrossRef]

${ }^{23}$ Ati-Hellal, M. E.; Hellal, F.; Hedhili, A. Application of Plackett-Burman and Doehlert designs for optimization of selenium analysis in plasma with electrothermal atomic absorption spectrometry. Clinical Biochemistry 2014, 47, 95. [CrossRef] [Pubmed]

${ }^{24}$ Almeida, J. S.; Anunciação, T. A.; Brandão, G. C.; Dantas, A. F.; Lemos, V. A.; Teixeira, L. S. G. Ultrasound-assisted single-drop microextraction for the determination of cadmium in vegetable oils using highresolution continuum source electrothermal atomic absorption spectrometry. Spectrochimica Acta Part B 2015, 107, 159. [CrossRef]

${ }^{25}$ Lopez Guerrero, M. M.; Siles Cordero, M. T.; Vereda Alonso, E.; Cano Pavón, J. M.; García de Torres, A. High resolution continuum source atomic absorption spectrometry and solid phase extraction for the simultaneous separation/preconcentration and sequential monitoring of $\mathrm{Sb}, \mathrm{Bi}, \mathrm{Sn}$ and $\mathrm{Hg}$ in low concentrations. Journal of Analytical Atomic Spectrometry 2015, 30, 1169. [CrossRef]

${ }^{26}$ Frizzarin, R. M.; Portugal, L. A.; Estela, J. M.; Rocha, F. R. P.; Cerdà, V. On-line lab-insyringe cloud point extraction for the spectrophotometric determination of antimony. Talanta 2016, 148, 694. [CrossRef] [Pubmed]

${ }^{27}$ Morales, R.; Ortiz, M. C.; Sarabia, L. A.; Sánchez, M. S. D-optimal designs and N-way techniques to determine sulfathiazole in milk by molecular fluorescence spectroscopy. Analytica Chimica Acta 2011, 707, 38. [CrossRef] [PubMed]

${ }^{28}$ Portugal, L. A.; Ferrer, L.; Serra, A. M.; Silva, D. G.; Ferreira, S. L. C.; Cerda, V. J. A non- 
chromatographic automated system for antimony speciation in natural water exploiting multisyringe flow injection analysis coupled with online hydride generation atomic fluorescence spectrometry. Journal of Analytical Atomic Spectrometry 2015, 30, 1133. [CrossRef]

${ }^{29}$ Kamarei, F.; Ebrahimzadeh, H.; Yamini, Y. Optimization of ultrasound-assisted emulsification microextraction with solidification of floating organic droplet followed by high performance liquid chromatography for the analysis of phthalate esters in cosmetic and environmental water samples. Microchemical Journal 2011, 99, 26. [CrossRef]

${ }^{30}$ Meinhart, A. D.; Ballus, C. A.; Bruns, R. E.; Pallone, J. A. L.; Godoy, H. T. Chemometrics optimization of carbohydrate separations in six food matrices by micellar electrokinetic chromatography with anionic surfactant. Talanta 2011, 85, 237. [CrossRef] [PubMed]

${ }^{31}$ Martínez-Mejía, M. J.; Rath, S. Use of experimental design to optimize a triplepotential waveform to develop a method for the determination of streptomycin and dihydrostreptomycin in pharmaceutical veterinary dosage forms by HPLC-PAD. Journal of Pharmaceutical and Biomedical Analysis 2015, 104, 81. [CrossRef] [PubMed]

${ }^{32}$ Benevides, C. M. J.; Bezerra, M. A.; Pereira, P. A. P.; Andrade, J. B. HSSPME/ GC-MS analysis of $\mathrm{VOC}$ and multivariate techniques applied to the discrimination of Brazilian varieties of mango. American Journal of Analytical Chemistry 2014, 5, 157. [CrossRef]

${ }^{33}$ Martendal, E.; Budziak, D.; Carasek, E. Application of fractional factorial experimental and Box-Behnken designs for optimization of single-drop microextraction of 2,4,6-trichloroanisole and 2,4,6tribromoanisole from wine samples. Journal of Chromatography A 2007, 1148, 131. [CrossRef] [PubMed]

${ }^{34}$ Merib, J.; Nardini, G.; Carasek, E. Use of Doehlert design in the optimization of extraction conditions in the determination of organochlorine pesticides in bovine milk samples by HS-SPME. Analytical Methods 2014, 6, 3254. [CrossRef]

${ }^{35}$ Costa, S. S. L.; Pereira, A. C. L.; Passos, E. A.; Alves, J. D. H.; Garcia, C. A. B.; Araújo, R. G. O. Multivariate optimization of an analytical method for the analysis of dog and cat foods by ICP OES. Talanta 2013, 108, 157. [CrossRef] [PubMed]

${ }^{36}$ Escudero, L. A.; Pacheco, P. H.; Gasquez, J. A.; Salonia, J. A. Development of a FI-HG-ICPOES solid phase preconcentration system for inorganic selenium speciation in Argentinean beverages. Food Chemistry 2015, 169, 73. [CrossRef] [Pubmed]

${ }^{37}$ Nomngongo, P. N.; Ngila, J. C. Determination of trace $\mathrm{Cd}, \mathrm{Cu}, \mathrm{Fe}, \mathrm{Pb}$ and $\mathrm{Zn}$ in diesel and gasoline by inductively coupled plasma mass spectrometry after sample clean up with hollow fiber solid phase microextraction system. Spectrochimica Acta Part B 2014, 98, 54. [CrossRef]

${ }^{38}$ Candela, A. M.; Coello, J.; Palet, C. Doehlert experimental design as a tool to study liquidliquid systems for the recovery of Uranium (VI) traces. Separation and Purification Technology 2013, 118, 399. [CrossRef]

${ }^{39}$ Asfaw, A.; Beauchemin, D. Combination of a multimode sample introduction system with a pre-evaporation tube to improve multi-element analysis by ICP-OES. Journal of Analytical Atomic Spectrometry 2012, 27, 80. [CrossRef]

${ }^{40}$ Kadar, A.; Noël, L.; Chekri, R.; Vastel, C.; Millour, S.; Guérin, T. Optimization of ICP-MS collision/reaction cell conditions for the determination of elements likely to be interfered ( $\mathrm{V}, \mathrm{Cr}, \mathrm{Fe}, \mathrm{Co}, \mathrm{Ni}, \mathrm{As}$ and $\mathrm{Se})$ in foodstuffs. Talanta 2011, 85, 2605. [CrossRef] [PubMed]

${ }^{41}$ Lopes, W. L.; Santelli, R. E.; Oliveira, E. P.; Carvalho, M. F.; Bezerra, M. A. Application of multivariate techniques in the optimization of a procedure for the determination of bioavailable concentrations of Se and As in estuarine sediments by ICP OES using a concomitant metals analyzer as a hydride 
generator. Talanta 2009, 79, 1276. [CrossRef] [PubMed]

${ }^{42}$ Nomngongo, P. N.; Ngila, J. C.; Msagati, T. A. M.; Moodley, B. Chemometric optimization of hollow fiber-liquid phase microextraction for preconcentration of trace elements in diesel and gasoline prior to their ICP-OES determination. Microchemical Journal 2014, 114, 141. [CrossRef]

${ }^{43}$ Bispo, M. S.; Morte, E. S. B.; Korn, M. G. A.; Teixeira, L. S. G.; Korn, M.; Costa, A. C. S. Determination of $\mathrm{Pb}$ in river water samples by inductively coupled plasma optical emission spectrometry after ultrasoundassisted co-precipitation with manganese dioxide. Spectrochimica Acta Part B 2005, 60, 653. [CrossRef]

${ }^{44}$ Gorla, F. A.; Duarte, E. H.; Sartori, E. R.; Tarley, C. R. T. Electrochemical study for the simultaneous determination of phenolic compounds and emerging pollutant using an electroanalytical sensing system based on carbon nanotubes/surfactant and multivariate approach in the optimization. Microchemical Journal 2016, 124, 65. [CrossRef]

${ }^{45}$ Trindade, J. M.; Martiniano, L. C.; Gonçalves, V. R. A.; Souza, A. G.; Marques, A. L. B.; Baugis, G. L.; Fonseca, T. C. O.; Song, C.; Zhang, J.; Marques, E. P. Anodic stripping voltammetry coupled with design of experiments for simultaneous determination of $\mathrm{Zn}^{+2}, \mathrm{Cu}^{+2}, \mathrm{~Pb}^{+2}$, and $\mathrm{Cd}^{+2}$ in gasoline. Fuel 2012, 91, 26. [CrossRef]

${ }^{46}$ Hajian, R.; Ensafi, A. A. Determination of aflatoxins B1 and B2 by adsorptive cathodic stripping voltammetry in groundnut. Food Chemistry 2009, 115, 1034. [CrossRef]

${ }^{47}$ Cuéllar, M.; Pfaffen, V.; Ortiz, P. I. Application of multi-factorial experimental design to successfully model and optimize inorganic chromium speciation by square wave voltammetry. Journal of Electroanalytical Chemistry 2016, 765, 37. [CrossRef]

${ }^{48}$ Hevia, D.; Botas, C.; Sainz, R. M.; Quiros, I.; Blanco, D.; Tand, D. X.; Gomez-Cordoves, C.; Mayo, J. C. Development and validation of new methods for the determination of melatonin and its oxidative metabolites by high performance liquid chromatography and capillary electrophoresis, using multivariate optimization. Journal of Chromatography $A$ 2010, 1217, 1368. [CrossRef] [PubMed]

${ }^{49}$ Ballus, C. A.; Meinhart, A. D.; Bruns, R. E.; Godoy, H. T. Use of multivariate statistical techniques to optimize the simultaneous separation of 13 phenolic compounds from extra-virgin olive oil by capillary electrophoresis. Talanta 2011, 83, 1181. [CrossRef] [PubMed]

${ }^{50}$ Fukuji, T. S.; Tonin, F. G.; Tavares, M. F. M. Optimization of a method for determination of phenolic acids in exotic fruits by capillary electrophoresis. Journal of Pharmaceutical and Biomedical Analysis 2010, 51, 430. [CrossRef] [Pubmed]

${ }^{51}$ Pellerano, R. G.; Romero, C. H.; Acevedo, H. A.; Vazquez, F. A. Un método de bajo costo para la determinación de cobre a nivel de vestigios en matrices de interés ambiental por Espectrofotometría en Fase Sólida (EFS). Química Nova 2007, 30, 2020. [CrossRef]

${ }^{52}$ Santos, Q. O.; Bezerra, M. A.; Lima, G. F.; Diniz, K. M.; Sagatelli, M. G.; Germiniano, T. O.; Santos, V. S.; Tarley, C. R. T. Synthesis, characterization and application of ion imprinted poly(vinylimidazole) for zinc ion extraction/preconcentration with FAAS determination. Química Nova 2014, 37, 63. [CrossRef]

${ }^{53}$ Silveira, G.; Tarley, C. R. T. Determinação turbidimétrica do antidepressivo amitriptilina em sistema FIA explorando a formação do par iônico com lauril sulfato de sódio. Química Nova 2008, 31, 1653. [CrossRef]

${ }^{54}$ Vicentini, F. C.; Figueiredo-Filho, L. C. S.; Janegitz, B. C.; Santiago, A.; Pereira-Filho, E. R.; Fatibello-Filho, O. Planejamento fatorial e superfície de resposta: otimização de um método voltamétrico para a determinação de $\mathrm{Ag}(\mathrm{I})$ empregando um eletrodo de pasta de nanotubos de carbono. Química Nova 2011, 34, 825. [CrossRef]

${ }^{55}$ Garda, J.; Badiale-Furlong, E. Otimização de metodologia para derivação de desoxinivalenol através de planejamento 
experimental. Química Nova 2008, 31, 270. [CrossRef]

${ }^{56}$ Kowalski, C. H.; Augusto, F. Extração de bifenilas policloradas de amostras de leite materno: otimização univariada versus planejamento experimental. Química Nova 2013, 36, 468. [CrossRef]

57 Leardi, R.; Experimental design in chemistry: A tutorial. Analytica Chimica Acta 2009, 652, 161. [CrossRef] [PubMed]

58 Armstrong, N. A.; Pharmaceutical experimental design and interpretation, 2nd ed., Taylor \& Francis: London, 2006.

59 Pimentel, M. F.; Neto, B. B. Calibração: Uma Revisão para Químicos Analíticos. Química Nova 1996, 19, 268. [Link]

${ }^{60}$ Santos, F. M.; Simões, J. C.; Silva, J. R. A.; Barthus, R. C.; Poppi, R. J.; Amaral, A. C. S. Otimização das condições de extração de saponinas em Ampelozizyphus amazonicus usando planejamento experimental e metodologia de superfície de resposta. Química Nova 2011, 34, 1629. [CrossRef]

${ }^{61}$ Callao, M. P. Multivariate experimental design in environmental analysis. TrAC, Trends in Analytical Chemistry 2014, 62, 86. [CrossRef]

62 Teófilo, R. F.; Ferreira, M. M. C. Quimiometria II: planilhas eletrônicas para cálculos de planejamentos experimentais, um tutorial. Química Nova 2006, 29, 338. [CrossRef]

${ }^{63}$ Santelli, R. E.; Bezerra, M. A.; Freire, A. S.; Oliveira, E. P.; Carvalho, M. F. B. Non-volatile vanadium determination in petroleum condensate, diesel and gasoline prepared as detergent emulsions using GF AAS. Fuel 2008, 87, 1617. [CrossRef]

${ }^{64}$ Barros, J. M.; Bezerra, M. A.; Valasques, G. S.; Nascimento Júnior, B. B.; Souza, A. S.; Aragão, N. M. Multivariate optimization of an ultrasound-assisted extraction procedure for $\mathrm{Cu}, \mathrm{Mn}, \mathrm{Ni}$ and $\mathrm{Zn}$ determination in ration to chickens. Anais da Academia Brasileira de Ciências 2013, 85, 891. [CrossRef] [PubMed]

${ }^{65}$ Breitkreitz, M. C.; Souza, A. M.; Poppi, R. J. Experimento didático de quimiometria para planejamento de experimentos: Avaliação das condições experimentais na determinação espectrofotométrica de ferro com o-fenantrolina. Um tutorial, Parte III. Química Nova 2014, 37, 564. [CrossRef]

${ }^{66}$ Nunes, C. A.; Alvarenga, V. O.; Sant'Ana, A. S.; Santos, J. S.; Granato, D. The use of statistical software in food Science and technology: Advantages, limitations and misuses. Food Research International 2015, 75, 270. [CrossRef]

${ }^{67}$ Ramos, G. R.; Álvarez-Coque. M. C. G. Quimiometría, Editorial Sintesis: Madri, 2014.

${ }^{68}$ Sítio da Chemkeys. Disponível em: <http://chemkeys.com/br/2009/08/07/tutori ais-e-programas-computacionais/> Acesso em: 16 março 2017.

${ }^{69}$ Sítio da Chemoface. Disponível em: <http://ufla.br/chemoface/> Acesso em: 16 março 2017.

${ }^{70}$ Sítio da Equipe Estatcamp. Software Action. Disponível em <http://www.portalaction.combr/> Acesso em: 16 março 2017. 